Yan Gao • Selda Oterkus

\title{
Ordinary state-based peridynamic modelling for fully coupled thermoelastic problems
}

Received: 30 December 2017 / Accepted: 14 June 2018 / Published online: 9 July 2018

(C) The Author(s) 2018

\begin{abstract}
An ordinary state-based peridynamic model is developed for transient fully coupled thermoelastic problems. By adopting an integral form instead of spatial derivatives in the equation of motion, the developed model is still valid at discontinuities. In addition, the ordinary state-based peridynamic model eliminates the limitation on Poisson's ratio which exists in bond-based peridynamics. Interactions between thermal and structural responses are also considered by including the coupling terms in the formulations. These formulations are also cast into their non-dimensional forms. Validation of the new model is conducted by solving some benchmark problems and comparing them with other numerical solutions. Thin plate and block under shockloading conditions are investigated. Good agreements are obtained by comparing the thermal and mechanical responses with those obtained from boundary element method and finite element solutions. Subsequently, a three-point bending test simulation is conducted by allowing crack propagation. Then a crack propagation for a plate with a pre-existing crack is investigated under pressure shock-loading condition. Finally, a numerical simulation based on the Kalthoff experiment is conducted in a fully coupled manner. The crack propagation processes and the temperature evolutions are presented. In conclusion, the present model is suitable for modelling thermoelastic problems in which discontinuities exist and coupling effects cannot be neglected.
\end{abstract}

Keywords State-based peridynamics $\cdot$ Fully coupled $\cdot$ Thermoelasticity $\cdot$ Crack propagation

\section{List of symbols}

\begin{tabular}{ll}
$A$ & Cross-sectional area for one-dimensional problems $\left(\mathrm{m}^{2}\right)$ \\
$c_{\mathrm{V}}$ & Specific heat capacity under constant volume $[\mathrm{J} /(\mathrm{kg} \mathrm{K})]$ \\
$E$ & Young's modulus (Pa) \\
$G_{\mathrm{c}}$ & Critical energy release rate $\left(\mathrm{J} / \mathrm{m}^{2}\right)$ \\
$h$ & Thickness for two-dimensional problems $(\mathrm{m})$ \\
$K_{\theta}$ & Bulk modulus $(\mathrm{GPa})$ \\
$k_{\mathrm{T}}$ & Thermal conductivity $[\mathrm{W} /(\mathrm{mK})]$ \\
$\mathbf{u}(\mathbf{x}, t)$ & Displacement of point $\mathbf{x}$ at time $t(\mathrm{~m})$ \\
$\dot{\mathbf{u}}(\mathbf{x}, t)$ & Velocity of point $\mathbf{x}$ at time $t(\mathrm{~m} / \mathrm{s})$ \\
$\ddot{\mathbf{u}}(\mathbf{x}, t)$ & Acceleration of point $\mathbf{x}$ at time $t\left(\mathrm{~m} / \mathrm{s}^{2}\right)$ \\
$u_{x}(\mathbf{x}, t)$ & Scalar value of horizontal displacement of point $\mathbf{x}$ at time $t(\mathrm{~m})$ \\
$\alpha$ & Linear thermal expansion coefficient $\left(\mathrm{K}^{-1}\right)$ \\
$\beta_{\mathrm{cl}}$ & Thermal modulus in classical mechanics $(\mathrm{Pa} / \mathrm{K})$ \\
\hline
\end{tabular}

Communicated by Francesco dell'Isola.

Y. Gao $\cdot$ S. Oterkus $(\varangle)$

Department of Naval Architecture, Ocean and Marine Engineering, University of Strathclyde, Glasgow, UK

E-mail: selda.oterkus@strath.ac.uk 


$\begin{array}{ll}\Theta_{0} & \text { Reference temperature }(\mathrm{K}) \\ \lambda, \mu & \text { Lamé constants }(\mathrm{GPa}) \\ v & \text { Poisson's ratio } \\ \rho & \text { Density }\left(\mathrm{kg} / \mathrm{m}^{3}\right) \\ V^{\prime} & \text { Volume of point } \mathbf{x}^{\prime}\left(\mathrm{m}^{3}\right) \\ \Theta(\mathbf{x}, t) & \text { Absolute temperature of point } \mathbf{x} \text { at time } t(\mathrm{~K}) \\ T(\mathbf{x}, t) & \text { Temperature change of point } \mathbf{x} \text { at time } t(\mathrm{~K}) \\ \dot{T}(\mathbf{x}, t) & \text { Time rate of temperature change of point } \mathbf{x} \text { at time } t(\mathrm{~K} / \mathrm{s}) \\ u_{y}(\mathbf{x}, t) & \text { Scalar value of vertical displacement of point } \mathbf{x} \text { at time } t(\mathrm{~m})\end{array}$

\section{Introduction}

Due to the development of aerospace and mechanical industries in recent years, mechanical and thermal shock loadings become typical and important loading types. For example, the gas turbine engine casing of an aircraft can experience a temperature rise as high as $1700^{\circ} \mathrm{C}$ within the extremely short period [1]. In the analyses with such loading conditions, the thermomechanical coupling effects often play a crucial role and as a consequence they should be considered both in thermal and in structural fields [2]. Not only the effect of temperature on deformation, but also the effect of deformation on temperature field is non-negligible. Therefore, fully coupled thermoelasticity analyses are necessary when dealing with such types of problems.

The basic theory of linear coupled thermoelasticity is well understood and fully developed for many years. Biot [3] first introduced a coupling item in heat conduction equation to solve the coupled problem of thermoelasticity. Later, Herrmann [4] generalized Biot's principle to a three-dimensional anisotropic body. Recently, Jabbari et al. [5,6] gave exact equations for classical coupled thermoelasticity in cylindrical and spherical coordinates. Although some analytical solutions are provided for some simple problems, many complex problems have not been completely solved with analytical methods [7]. Therefore, numerical methods such as finite element method (FEM) and boundary element method (BEM) have been widely applied to get approximate solutions [8]. For example, Cannarozzi and Ubertini [9] conducted FEM analyses for linear coupled quasi-static thermoelastic problems with a mixed variation method. Displacement and temperature acted as primary variables in their research. On the other hand, stress and heat flux acted as dual variables which were also involved directly in their analysis. Tehrani and Eslami [10] studied the coupling coefficients and relaxation time effects on thermal and elastic wave motion by using BEM. When fracture is involved in the fully coupled analyses, temperature distribution around the crack tips becomes a major concern. The high energy concentration around a moving crack produces high amount of heat energy and results in unneglectable temperature increase. Atkinson and Craster [11] deduced some simple and asymptotic temperature distributions for the region near crack tips during crack propagation. Weichert and Schönert [12] investigated the temperature near the crack tips in brittle materials with very small plastic zones and high crack velocities. The crack tips were simulated as heat generation sources, and consequently, the temperature distributions were predicted. Experimental study conducted by Bhalla et al. [13] estimated the temperature distribution near the crack tips. A temperature increase was observed in their experiments. Miehe et al. [14] presented a continuum phase-field model for brittle fracture in thermoelasticity. A bending numerical simulation test considering the crack growth and dissipative heat generation was provided, and its corresponding temperature field was discussed.

When discontinuity is involved in thermoelastic problems, the aforementioned numerical simulation methods based on the classical mechanic theory predict unbounded stresses and energy densities. Even for linear elastic fracture mechanics (LEFM) and dislocation dynamics, supplemental constitutive equations are needed to determine the motion of a dislocation. On the contrary, peridynamics (PD) [15-20] is a non-local theory that includes damage as part of the material response. The formulation in peridynamics is in an integral-differential form, instead of spatial derivation equations in classical continuum mechanics. As a consequence, the PD equations still remain valid where crack or discontinues merge [22]. Therefore, PD theory is especially suitable for problems with discontinues; thus, it is adopted in this paper. The crack nucleation and propagation have been investigated by many researchers in the realm of PD theory [23-38], but most of them are only in mechanical field. In thermal field, Oterkus et al. [39] derived the formulation of thermal diffusion with PD theory and utilized it to capture the fuel pellet cracking [40]. Bobaru and Duangpanya [41,42] studied the heat conduction in bodies with and without discontinuities in bond-based PD theory. In regard to the thermomechanics, fully coupled bond-based PD theory was formulated by Oterkus et al. [43], Oterkus [44], Madenci and Oterkus [45]. Further, they successfully applied their model to predict crack propagation [46,47]. However, the 


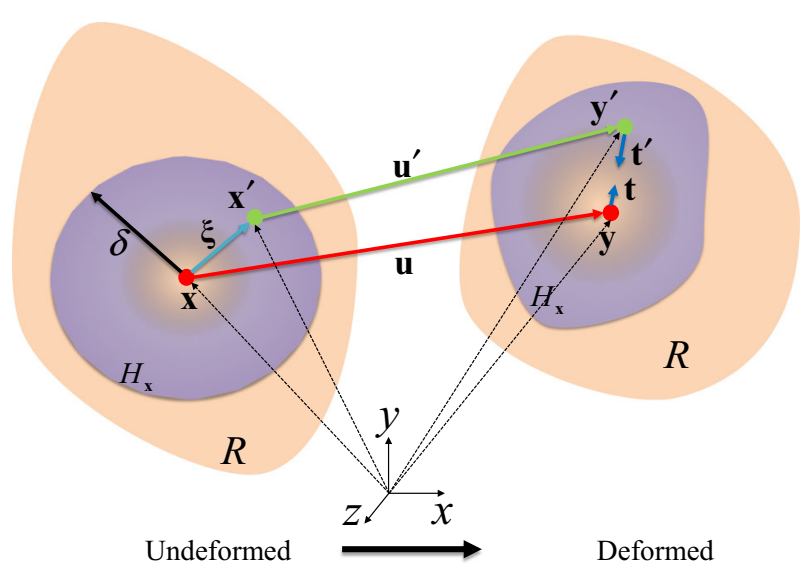

Fig. 1 Domain of interaction for a material point

bond-based PD model is limited to have a fixed Poisson's ratio, i.e. 1/3 for two-dimensional problems and 1/4 for three-dimensional problems [48]. Considering this constraint, a generalized PD model which eliminates the aforementioned limitations is necessary for thermomechanical problems.

The purpose of this paper is to develop a PD model overcoming the bond-based PD limitation on material properties for fully coupled thermoelastic problems. Therefore, ordinary state-based PD theory is employed and its formulations are derived in the realm of fully coupled thermomechanics. Then both dimensional and non-dimensional forms of the coupled equations in both deformation and thermal fields are provided and subsequently validated. Temperature changes and displacements of an isotropic thin plate and a block under various types of loading conditions are investigated. The validation is conducted via comparing the results with those from ANSYS and BEM solutions. The remarkable agreement indicates the capability of the proposed PD model to accurately predict the mechanical and thermal responses in a fully coupled manner. In the final part of this paper, crack propagation patterns are predicted for three-point bending test, Kalthoff problem. In addition, crack propagation for a plate with a pre-existing crack subjected to a pressure loading is conducted. The crack growth paths and temperature distributions are presented, and crack patterns are compared with the predictions from pure mechanical analysis. Hence, the effect of coupling term on crack growth is estimated and discussed.

\section{Fully coupled peridynamic thermomechanics}

The peridynamics falls into the category of non-local theories, i.e. particles interact with each other within a horizon, $\delta$, as shown in Fig 1. Each material point is identified by its location represented by coordinate $\mathbf{x}$ in an undeformed state. The body region is $R$, and the interaction domain of material point $\mathbf{x}$ is denoted by $H_{\mathbf{x}}$. The other material points in $H_{\mathbf{x}}$, i.e. $\mathbf{x}^{\prime}$, are called the family members of $\mathbf{x}$. Furthermore, $\mathbf{y}$ and $\mathbf{y}^{\prime}$ represent the positions of $\mathbf{x}$ and $\mathbf{x}^{\prime}$ in a deformed configuration. In the deformed configuration, $\left(\mathbf{t}-\mathbf{t}^{\prime}\right)$ is the force function exerted from point $\mathbf{x}^{\prime}$ to point $\mathbf{x}$. Similarly, $\left(\mathbf{t}^{\prime}-\mathbf{t}\right)$ is the force function acting at material point $\mathbf{x}^{\prime}$ from point $\mathbf{x}$. In bond-based PD theory, the pairwise force functions are equal in magnitude and also parallel to their relative position in the deformed state, i.e. $\mathbf{t} / / \mathbf{t}^{\prime}$ and $|\mathbf{t}|=\left|\mathbf{t}^{\prime}\right|[48,49]$. Therefore, Poisson's ratio is $1 / 3$ for two-dimensional (2D) problems and $1 / 4$ for three-dimensional (3D) problems [15]. On the other hand, the state-based force functions are still parallel to their relative position in the deformed state, i.e. $\mathbf{t} / / \mathbf{t}^{\prime}$. But they do not have to be equal in magnitude in ordinary state-based PD model, i.e. $|\mathbf{t}|=\left|\mathbf{t}^{\prime}\right|$ or $|\mathbf{t}| \neq\left|\mathbf{t}^{\prime}\right|$. Thus, ordinary state-based formulation overcomes the limitation on Poisson's ratio. Hence, in this study ordinary state-based PD theory is adopted for fully coupled thermoelastic problems. Regarding the fully coupled thermomechanical analysis, the ordinary state-based PD model has not been explicitly provided, and only the expression in bond-based theory is available in the published literature $[43,44,50]$. Therefore, in this study, ordinary state-based fully coupled PD thermoelastic equations with explicit formulations of PD parameters are provided. Then these equations are cast into their corresponding non-dimensional forms. 
2.1 Peridynamic thermoelastic formulations

The general form of fully coupled thermoelasticity is provided by Oterkus et al. [43]. The equation of motion can be shown as

$$
\rho \ddot{\mathbf{u}}(\mathbf{x}, t)=\int_{H_{\mathbf{x}}}\left(\mathbf{t}\left(\mathbf{u}^{\prime}-\mathbf{u}, \mathbf{x}^{\prime}-\mathbf{x}, t\right)-\mathbf{t}^{\prime}\left(\mathbf{u}-\mathbf{u}^{\prime}, \mathbf{x}-\mathbf{x}^{\prime}, t\right)\right) \mathrm{d} V^{\prime}+\mathbf{b}(\mathbf{x}, t)
$$

The integration on the right-hand side of Eq. (1) represents the total PD force density acting on point $\mathbf{x}$. Thereby, $\mathbf{b}(\mathbf{x}, t)$ represents the volumetric body force at material point $\mathbf{x}$. In Eq. (1) $\mathbf{t}\left(\mathbf{u}^{\prime}-\mathbf{u}, \mathbf{x}^{\prime}-\mathbf{x}, t\right)$ and $\mathbf{t}^{\prime}\left(\mathbf{u}-\mathbf{u}^{\prime}, \mathbf{x}-\mathbf{x}^{\prime}, t\right)$ are PD force functions that belong to material point $\mathbf{x}$ and $\mathbf{x}^{\prime}$. The ordinary state-based expressions for PD force functions are presented as provided in Chapter 4 of [49].

$$
\mathbf{t}\left(\mathbf{u}^{\prime}-\mathbf{u}, \mathbf{x}^{\prime}-\mathbf{x}, t\right)=\frac{1}{2} B_{1} \frac{\mathbf{y}^{\prime}-\mathbf{y}}{\left|\mathbf{y}^{\prime}-\mathbf{y}\right|}
$$

and

$$
\mathbf{t}^{\prime}\left(\mathbf{u}-\mathbf{u}^{\prime}, \mathbf{x}-\mathbf{x}^{\prime}, t\right)=-\frac{1}{2} B_{2} \frac{\mathbf{y}^{\prime}-\mathbf{y}}{\left|\mathbf{y}^{\prime}-\mathbf{y}\right|}
$$

where $B_{1}$ and $B_{2}$ are PD auxiliary parameters; those can be defined as

$$
\begin{aligned}
& B_{1}=4 \delta \frac{d \Lambda}{\left|\mathbf{x}^{\prime}-\mathbf{x}\right|} a(\theta-n \alpha T)+4 \delta b(s-\alpha T) \\
& B_{2}=4 \delta \frac{d \Lambda}{\left|\mathbf{x}-\mathbf{x}^{\prime}\right|} a\left(\theta^{\prime}-n \alpha T^{\prime}\right)+4 \delta b\left(s-\alpha T^{\prime}\right)
\end{aligned}
$$

with

$$
n= \begin{cases}2 & \text { for } 2 \mathrm{D} \\ 3 & \text { for } 3 \mathrm{D}\end{cases}
$$

where $T$ represents the temperature change of point $\mathbf{x}$ with respect to reference temperature, $T=\Theta(\mathbf{x}, t)-\Theta_{0}$. Similarly, $T^{\prime}$ is the temperature change of point $\mathbf{x}^{\prime}$. The dilatations, $\theta$ for point $\mathbf{x}$ and $\theta^{\prime}$ for point $\mathbf{x}^{\prime}$, are defined as

$$
\begin{gathered}
\theta=\int_{H_{\mathbf{x}}} d \delta(s-\alpha T) \Lambda \mathrm{d} V^{\prime}+n \alpha T \\
\theta^{\prime}=\int_{H_{\mathbf{x}^{\prime}}} d \delta(s-\alpha T) \Lambda \mathrm{d} V+n \alpha T^{\prime}
\end{gathered}
$$

The PD bond stretch, $s$, between material points $\mathbf{x}$ and $\mathbf{x}^{\prime}$ can be defined as

$$
s=\frac{\left|\mathbf{y}^{\prime}-\mathbf{y}\right|-\left|\mathbf{x}^{\prime}-\mathbf{x}\right|}{\left|\mathbf{x}^{\prime}-\mathbf{x}\right|}
$$

The PD auxiliary parameter, $\Lambda$, is defined as

$$
\Lambda=\left(\frac{\mathbf{y}^{\prime}-\mathbf{y}}{\left|\mathbf{y}^{\prime}-\mathbf{y}\right|}\right) \cdot\left(\frac{\mathbf{x}^{\prime}-\mathbf{x}}{\left|\mathbf{x}^{\prime}-\mathbf{x}\right|}\right)
$$

The relationship between the PD material parameters, i.e. $a, b$ and $d$, and classical material parameters are listed as provided in Chapter 4 of [49];

$$
\begin{aligned}
& a=0, b=\frac{E}{2 A \delta^{3}}, d=\frac{1}{2 A \delta^{2}} \text { for } 1 \mathrm{D} \\
& a=\frac{1}{2}\left(K_{\theta}-2 \mu\right), b=\frac{6 \mu}{\pi h \delta^{4}}, d=\frac{2}{\pi h \delta^{3}} \text { for } 2 \mathrm{D} \\
& a=\frac{1}{2}\left(K_{\theta}-\frac{5}{3} \mu\right), b=\frac{15 \mu}{2 \pi \delta^{5}}, d=\frac{9}{4 \pi \delta^{4}} \text { for } 3 \mathrm{D}
\end{aligned}
$$


The equation of motion for ordinary state-based formulation including the effect of temperature change can be written as

$$
\rho \ddot{\mathbf{u}}(\mathbf{x}, t)=\int_{H_{\mathbf{x}}}\left[\frac{2 \delta d \Lambda}{\left|\mathbf{x}^{\prime}-\mathbf{x}\right|}\left(a\left(\theta+\theta^{\prime}\right)-n \alpha\left(T+T^{\prime}\right)\right)+4 \delta b\left(s-\alpha \frac{\left(T+T^{\prime}\right)}{2}\right)\right] \frac{\mathbf{y}^{\prime}-\mathbf{y}}{\left|\mathbf{y}^{\prime}-\mathbf{y}\right|} \mathrm{d} V^{\prime}+\mathbf{b}(\mathbf{x}, t)
$$

On the other hand, the heat conduction equation in the fully coupled thermomechanical PD model is $[43,44]$

$$
\rho c_{\mathrm{v}} \dot{T}(\mathbf{x}, t)=\int_{H_{\mathbf{x}}}\left(\kappa \frac{\Theta\left(\mathbf{x}^{\prime}, t\right)-\Theta(\mathbf{x}, t)}{\left|\mathbf{x}^{\prime}-\mathbf{x}\right|}-\Theta_{0} \beta\left(\mathbf{x}^{\prime}-\mathbf{x}\right) \dot{e}\left(\mathbf{x}^{\prime}-\mathbf{x}\right)\right) \mathrm{d} V^{\prime}+h_{s}(\mathbf{x}, t)
$$

where $h_{s}(\mathbf{x}, t)$ is the rate of heat generation per unit volume. In the above equation, $\kappa$ is defined as PD microconductivity with its definitions listed as $[37,39,43]$

$$
\begin{aligned}
\kappa & =\frac{2 k_{\mathrm{T}}}{A \delta^{2}} \text { for } 1 \mathrm{D} \\
\kappa & =\frac{6 k_{\mathrm{T}}}{\pi h \delta^{3}} \text { for } 2 \mathrm{D} \\
\kappa & =\frac{6 k_{\mathrm{T}}}{\pi \delta^{4}} \text { for } 3 \mathrm{D}
\end{aligned}
$$

The second term in Eq. (12) represents the deformation coupling effect on temperature. The time rate of change of stretch extension, $\dot{e}\left(\mathbf{x}^{\prime}-\mathbf{x}\right)$, can be defined as

$$
\dot{e}=\frac{\mathbf{y}^{\prime}-\mathbf{y}}{\left|\mathbf{y}^{\prime}-\mathbf{y}\right|} \cdot\left(\dot{\mathbf{u}^{\prime}}-\dot{\mathbf{u}}\right)
$$

The physical meaning and theory foundation of the PD thermal modulus are fully discussed by Oterkus et al. [43]. In this paper, the same derivation approach is adopted. The initial form of the ordinary state PD force function for point $\mathbf{x}$ is shown in Eqs. (2) and (4). In another form, being similar to the derivation conducted by Oterkus et al. [43], the PD force function can be divided into two parts as [43]

$$
\mathbf{t}=\mathbb{k} \cdot \mathbf{u}-\mathbf{B} T
$$

The first part on the right-hand side includes only the structural deformation, and the second part is related to temperature effect. In Eq. (15), $\mathbb{k}$ is called the modulus state [51]; the term $\mathbf{B} T$ represents the effects of thermal state on deformation.

Substituting the expression in Eq. (4) to Eq. (2) PD force function can be obtained as

$$
\mathbf{t}\left(\mathbf{u}^{\prime}-\mathbf{u}, \mathbf{x}^{\prime}-\mathbf{x}, t\right)=\left[2 \delta \frac{d \Lambda}{\left|\mathbf{x}^{\prime}-\mathbf{x}\right|} a(\theta-n \alpha T)+2 \delta b(s-\alpha T)\right] \frac{\mathbf{y}^{\prime}-\mathbf{y}}{\left|\mathbf{y}^{\prime}-\mathbf{y}\right|}
$$

By plugging the dilatation term in Eq. (7a) the PD force function becomes

$$
\mathbf{t}\left(\mathbf{u}^{\prime}-\mathbf{u}, \mathbf{x}^{\prime}-\mathbf{x}, t\right)=\left[2 \delta \frac{d \Lambda}{\left|\mathbf{x}^{\prime}-\mathbf{x}\right|} a\left(\int_{H_{\mathbf{x}}} d \delta(s-\alpha T) \Lambda \mathrm{d} V^{\prime}\right)+2 \delta b(s-\alpha T)\right] \frac{\mathbf{y}^{\prime}-\mathbf{y}}{\left|\mathbf{y}^{\prime}-\mathbf{y}\right|}
$$

After rewriting the PD force function by splitting into pure mechanical and thermal part Eq. (17) becomes

$$
\mathbf{t}=\left[\left(\frac{2 a \delta^{2} d^{2} \Lambda}{\left|\mathbf{x}^{\prime}-\mathbf{x}\right|} \int_{H_{\mathbf{x}}} s \Lambda \mathrm{d} V^{\prime}+2 \delta b s\right) \frac{\mathbf{y}^{\prime}-\mathbf{y}}{\left|\mathbf{y}^{\prime}-\mathbf{y}\right|}\right]-\left[\left(\frac{2 a \delta^{2} d^{2} \Lambda}{\left|\mathbf{x}^{\prime}-\mathbf{x}\right|} \int_{H_{\mathbf{x}}} \Lambda \mathrm{d} V^{\prime}+2 \delta b\right)\left(\alpha \frac{\mathbf{y}^{\prime}-\mathbf{y}}{\left|\mathbf{y}^{\prime}-\mathbf{y}\right|}\right)\right] T
$$


By comparing Eqs. (15) and (18), local thermal modulus of bond between $\mathbf{x}$ and $\mathbf{x}^{\prime}$ can be obtained as

$$
\beta=\left(\frac{2 a \delta^{2} d^{2} \Lambda}{\left|\mathbf{x}^{\prime}-\mathbf{x}\right|} \int_{H_{\mathbf{x}}} \Lambda \mathrm{d} V^{\prime}+2 \delta b\right) \alpha
$$

with $\mathbf{B}=\beta \frac{\mathbf{y}^{\prime}-\mathbf{y}}{\left|\mathbf{y}^{\prime}-\mathbf{y}\right|}$ [43]. Substituting the PD parameters provided in Eq. (10) as

$$
\begin{aligned}
& \beta=\left(\frac{2(0) \delta^{2}\left(\frac{1}{2 A \delta^{2}}\right)^{2} \Lambda}{\left|\mathbf{x}^{\prime}-\mathbf{x}\right|} \int_{H_{\mathbf{x}}} \Lambda \mathrm{d} V^{\prime}+2 \delta\left(\frac{E}{2 A \delta^{3}}\right)\right) \alpha \text { for 1D } \\
& \beta=\left(\frac{2\left(\frac{1}{2}\left(K_{\theta}-2 \mu\right) \delta^{2}\left(\frac{2}{\pi h \delta^{3}}\right)^{2} \Lambda\right.}{\left|\mathbf{x}^{\prime}-\mathbf{x}\right|} \int_{H_{\mathbf{x}}} \Lambda \mathrm{d} V^{\prime}+2 \delta\left(\frac{6 \mu}{\pi h \delta^{4}}\right)\right) \alpha \text { for 2D } \\
& \beta=\left(\frac{2\left(\frac{1}{2}\left(K_{\theta}-\frac{5}{3} \mu\right)\right) \delta^{2}\left(\frac{9}{4 \pi \delta^{4}}\right)^{2} \Lambda}{\left|\mathbf{x}^{\prime}-\mathbf{x}\right|} \int_{H_{\mathbf{x}}} \Lambda \mathrm{d} V^{\prime}+2 \delta\left(\frac{15 \mu}{2 \pi \delta^{5}}\right)\right) \alpha \text { for 3D }
\end{aligned}
$$

Then the explicit forms of the local thermal modulus, $\beta$, for different dimensions can be obtained as

$$
\begin{aligned}
& \beta=\frac{E \alpha}{A \delta^{2}} \text { for } 1 \mathrm{D} \\
& \beta=\frac{4 \alpha}{\pi h \delta^{3}}\left(\frac{K_{\theta}-2 \mu}{\pi h \delta} \frac{\Lambda}{\left|\mathbf{x}^{\prime}-\mathbf{x}\right|} \int_{H_{\mathbf{x}}} \Lambda \mathrm{d} V^{\prime}+3 \mu\right) \text { for 2D } \\
& \beta=\frac{3 \alpha}{\pi \delta^{4}}\left(\frac{\left(27 K_{\theta}-45 \mu\right)}{16 \pi \delta^{2}} \frac{\Lambda}{\left|\mathbf{x}^{\prime}-\mathbf{x}\right|} \int_{H_{\mathbf{x}}} \Lambda \mathrm{d} V^{\prime}+5 \mu\right) \text { for 3D }
\end{aligned}
$$

Furthermore, if the bond-based restriction, $a=0$, is applied as explained in Chapter 4 of [49], Eq. (21) will reduce to its bond-based form, where $\beta_{b}=2 \delta b \alpha$ or $\beta_{b}=1 / 2(c \alpha)$ with $c$ is the bond-based PD material constant [43]. The bond-based PD form of thermomechanical formulations is provided in Appendix A.

\subsection{Non-dimensional form of peridynamics thermoelastic formulations}

The governing equation can be put in a non-dimensional form by using non-dimensional variables [52]. Therefore, the fully coupled PD equations are cast into their non-dimensional forms by adopting the approach proposed by Sackman [53] and Oterkus et al. [43].

Regarding the heat conduction equation, the diffusivity is defined as the characteristic length/time quantity

$$
\gamma=\frac{k_{\mathrm{T}}}{\rho c_{\mathrm{v}}}=\frac{l^{* 2}}{t^{*}}
$$

For the equation of motion, the characteristic length/time is the elastic wave speed

$$
\tilde{a}=\sqrt{\frac{\lambda+2 \mu}{\rho}}=\frac{l^{*}}{t^{*}}
$$

where $\lambda$ and $\mu$ are Lame's constants. Combining the characteristic length/timescale leads to characteristic length and time as follows

$$
\begin{aligned}
& l^{*}=\gamma / \tilde{a} \\
& t^{*}=\gamma / \tilde{a}^{2}
\end{aligned}
$$


As explained in [43] following non-dimensional forms can be used

Length-related variables:

$$
x=\frac{\gamma}{\tilde{a}} \bar{x}, \delta=\frac{\gamma}{\tilde{a}} \bar{\delta}, A=\left(\frac{\gamma}{\tilde{a}}\right)^{2} \bar{A}, V=\left(\frac{\gamma}{\tilde{a}}\right)^{3} \bar{V}
$$

Displacement:

$$
u=\left(\frac{\gamma}{\tilde{a}}\right) \frac{\beta_{\mathrm{cl}} \theta_{o}}{(\lambda+2 \mu)} \bar{u}
$$

Stretch:

$$
s=\frac{\beta_{\mathrm{cl}} \theta_{o}}{(\lambda+2 \mu)} \bar{s}
$$

Time:

$$
t=\left(\frac{\gamma}{\tilde{a}^{2}}\right) \bar{t}
$$

Velocity-related variables:

$$
v=\frac{\beta_{\mathrm{cl}} \theta_{o}}{(\lambda+2 \mu)} \tilde{a} \bar{v} \text { and } \dot{e}=\frac{\beta_{\mathrm{cl}} \theta_{o}}{(\lambda+2 \mu)} \tilde{a} \overline{\dot{e}}
$$

And the temperature:

$$
T=\theta_{o} \bar{T}
$$

By using the above non-dimensional parameters and substituting the peridynamic parameters listed in Eqs. (10), (13) and (21) into Eqs. (11) and (12), the non-dimensional form of the fully coupled equations can be achieved by utilizing the non-dimensional parameters given in Eqs. (22-30) as 1D analysis

$$
\begin{aligned}
& \overline{\dot{T}}=\frac{2}{\bar{A} \bar{\delta}^{2}} \int_{H_{\overline{\mathbf{x}}}}\left(\frac{\bar{T}^{\prime}-\bar{T}}{\left|\overline{\mathbf{x}}^{\prime}-\overline{\mathbf{x}}\right|}-\epsilon \frac{\bar{e}}{2}\right) \mathrm{d} \bar{V}^{\prime}+\bar{h}_{s} \text { for } 1 \mathrm{D} \\
& \overline{\mathbf{\mathbf { u }}}=\frac{2}{\bar{A} \bar{\delta}^{2}} \int_{H_{\overline{\mathbf{x}}}}\left(\bar{s}-\frac{\bar{T}+\bar{T}^{\prime}}{2}\right) \frac{\overline{\mathbf{y}}^{\prime}-\overline{\mathbf{y}}}{\left|\overline{\mathbf{y}}^{\prime}-\overline{\mathbf{y}}\right|} \mathrm{d} \bar{V}^{\prime}+\overline{\mathbf{b}} \text { for } 1 \mathrm{D}
\end{aligned}
$$

2D analysis

$$
\begin{aligned}
& \overline{\dot{T}}=\frac{6}{\pi \bar{h} \bar{\delta}^{3}} \int_{H_{\overline{\mathbf{x}}}}\left(\frac{\bar{T}^{\prime}-\bar{T}}{\left|\overline{\mathbf{x}}^{\prime}-\overline{\mathbf{x}}\right|}-\frac{\epsilon \overline{\dot{e}}}{1+v}\left[1-v+\left(v-\frac{1}{3}\right) \frac{\Lambda}{\pi \bar{h} \bar{\delta}\left|\overline{\mathbf{x}}^{\prime}-\overline{\mathbf{x}}\right|} \int_{H_{\overline{\mathbf{x}}}} \Lambda \mathrm{d} V^{\prime}\right]\right) \mathrm{d} \bar{V}^{\prime}+\bar{h}_{s} \text { for } 2 \mathrm{D} \\
& \overline{\mathbf{u}}=\frac{2}{\pi \bar{h}^{3} \bar{\delta}^{3}(1+v)} \int_{H_{\overline{\mathbf{x}}}}\left[\frac{3 v-1}{\pi \bar{h} \bar{\delta}\left|\overline{\mathbf{x}}^{\prime}-\overline{\mathbf{x}}\right|} \Lambda\left(\bar{\theta}+\bar{\theta}^{\prime}\right)+6(1-v)\left((1+v) \bar{s}-\frac{\bar{T}+\bar{T}^{\prime}}{2}\right)\right] \frac{\overline{\mathbf{y}}^{\prime}-\overline{\mathbf{y}}}{\left|\overline{\mathbf{y}} \overline{\mathbf{y}}^{\prime}-\overline{\mathbf{y}}\right|} \mathrm{d} \bar{V}^{\prime}+\overline{\mathbf{b}} \text { for } 2 \mathrm{D}
\end{aligned}
$$

with

$$
\bar{\theta}=\int_{H_{\overline{\mathbf{x}}}}[(1+v) \bar{s}-\bar{T}] \Lambda \mathrm{d} \bar{V}^{\prime} \text { for } 2 \mathrm{D}
$$

3D analysis

$$
\begin{aligned}
\overline{\dot{T}}= & \frac{6}{\pi \bar{\delta}^{4}} \int_{H_{\overline{\mathbf{x}}}}\left(\frac{\bar{T}^{\prime}-\bar{T}}{\left|\overline{\mathbf{x}}^{\prime}-\overline{\mathbf{x}}\right|}-\frac{\epsilon \overline{\dot{e}}}{16(1+v)}\left[20(1-2 v)+27\left(v-\frac{1}{4}\right) \frac{\Lambda}{\left|\overline{\mathbf{x}}^{\prime}-\overline{\mathbf{x}}\right| \pi \bar{\delta}^{2}} \int_{H_{\overline{\mathbf{x}}}} \Lambda \mathrm{d} V^{\prime}\right]\right) \mathrm{d} \bar{V}^{\prime}+\bar{h}_{s} \text { for 3D } \\
\overline{\mathbf{u}}= & \frac{3}{(1+v) \pi \bar{\delta}^{4}} \int_{H_{\mathbf{x}}}\left[\frac{27}{8}\left(v-\frac{1}{4}\right) \frac{\Lambda\left(\bar{\theta}+\bar{\theta}^{\prime}\right)}{\pi \bar{\delta}^{2}|\overline{\boldsymbol{\xi}}|}\right. \\
& \left.+5(1-2 v)\left(\frac{1+v}{1-v}-\frac{\bar{T}+\bar{T}^{\prime}}{2}\right)\right] \frac{\overline{\mathbf{y}}^{\prime}-\overline{\mathbf{y}}}{\left|\overline{\mathbf{y}}^{\prime}-\overline{\mathbf{y}}\right|} \mathrm{d} \bar{V}^{\prime}+\overline{\mathbf{b}} \text { for 3D }
\end{aligned}
$$


with

$$
\bar{\theta}=\int_{H_{\overline{\mathbf{x}}}}\left(\frac{1+v}{1-v} \bar{s}-\bar{T}\right) \Lambda \mathrm{d} \bar{V}^{\prime} \text { for 3D }
$$

In the above equations, the non-dimensional variables are denoted with an over score. The parameters $\overline{\dot{T}}$ and $\overline{\dot{T}}^{\prime}$ are the rate of temperature changes at material point $\overline{\mathbf{x}}$ and $\overline{\mathbf{x}}^{\prime}$, respectively. The non-dimensional coupling coefficient, $\epsilon$, measures the strength of coupling effect on temperature distribution due to deformation. It can be defined as [43]

$$
\epsilon=\frac{\beta_{\mathrm{cl}}^{2} \Theta_{0}}{\rho c_{\mathrm{v}}(\lambda+2 \mu)}
$$

\subsection{Peridynamic failure criteria}

Since PD equations are formulated without any spatial derivatives, PD theory is suitable to be applied for failure prediction. Once the stretch between material points exceeds the critical stretch value, $s_{\mathrm{c}}$, the bond will be broken and will be removed permanently. At the same time, the force between these two points becomes zero. The critical stretch value for bond failure is related to the critical energy release rate $G_{\mathrm{c}}$ as provided in Chapter 6 of [49];

$$
s_{\mathrm{C}}=\left\{\begin{array}{l}
\sqrt{\frac{G_{\mathrm{c}}}{\left(3 \mu+\left(\frac{3}{4}\right)^{4}\left(K_{\theta}-2 \mu\right)\right) \delta}} \text { for } 3 \mathrm{D} \\
\sqrt{\frac{G_{\mathrm{c}}}{\left(\frac{6}{\pi} \mu+\frac{16}{9 \pi^{2}}\left(K_{\theta}-2 \mu\right)\right) \delta}} \text { for } 2 \mathrm{D}
\end{array}\right.
$$

A history-dependent damage function $\chi\left(\mathbf{x}, \mathbf{x}^{\prime}, t\right)$ is implemented for each interaction between the material points. The value of the function $\chi\left(\mathbf{x}, \mathbf{x}^{\prime}, t\right)$ will be set to be zero when the bond is broken.

$$
\chi\left(\mathbf{x}, \mathbf{x}^{\prime}, t\right)=\left\{\begin{array}{l}
1,\left(s-\alpha \frac{T+T^{\prime}}{2}\right)<s_{\mathrm{c}} \\
0,\left(s-\alpha \frac{T+T^{\prime}}{2}\right) \geq s_{\mathrm{c}}
\end{array}\right.
$$

The local damage at a point represents the weighted ratio of the number of the broken interactions to the total number of interaction. Therefore, crack propagation path can be presented by the local damage value as [48];

$$
\varphi(\mathbf{x}, t)=1-\frac{\int_{H_{\mathbf{x}}} \chi\left(\mathbf{x}, \mathbf{x}^{\prime}, t\right) \mathrm{d} V^{\prime}}{\int_{H_{\mathbf{x}}} \mathrm{d} V^{\prime}}
$$

\subsection{Numerical implementation}

The developed PD thermomechanical model is discretized for numerical implementation. Thus, the discretized form of equation of motion provided in Eq. (11) can be written as

$$
\begin{aligned}
\rho \ddot{\mathbf{u}}\left(\mathbf{x}_{i}, t\right)= & \left(\sum_{j=1}^{N_{i}}\left[\frac{2 \delta d \Lambda}{\left|\mathbf{x}_{j}-\mathbf{x}_{i}\right|}\left(a\left(\theta_{i}+\theta_{j}\right)-n \alpha\left(T_{i}+T_{j}\right)\right)+4 \delta b\left(s_{i j}-\alpha \frac{\left(T_{i}+T_{j}\right)}{2}\right)\right] \frac{\mathbf{y}_{j}-\mathbf{y}_{i}}{\left|\mathbf{y}_{j}-\mathbf{y}_{i}\right|} V_{j}\right) \\
& +\mathbf{b}\left(\mathbf{x}_{i}, t\right)
\end{aligned}
$$

where $i$ represents the point of interest, and $j$ represents the family members of point $i$.

The discretized form of dilatation provided in Eq. (7a) becomes

$$
\theta_{i}=\left(\sum_{j=1}^{N_{i}} d \delta\left(s_{i j}-\alpha T_{i}\right) \Lambda V_{j}\right)+n \alpha T_{i}
$$


Here, $N_{i}$ represents the number of family members for point $i$.

On the other hand, the discretized form heat equation provided in Eq. (12) can be written as

$$
\rho c_{\mathrm{v}} \dot{T}\left(\mathbf{x}_{i}, t\right)=\left(\sum_{j=1}^{N_{i}}\left(\kappa \frac{\Theta\left(\mathbf{x}_{j}, t\right)-\Theta\left(\mathbf{x}_{i}, t\right)}{\left|\mathbf{x}_{j}-\mathbf{x}_{i}\right|}-\Theta_{0} \beta\left(\mathbf{x}_{j}-\mathbf{x}_{i}\right) \dot{e}\left(\mathbf{x}_{j}-\mathbf{x}_{i}\right)\right) V_{j}\right)+h_{s}\left(\mathbf{x}_{i}, t\right)
$$

The local damage at point $i$ provided in Eq.(37) can be written as [49]

$$
\varphi\left(\mathbf{x}_{i}, t\right)=1-\frac{\sum_{j=1}^{N_{i}} \chi\left(\mathbf{x}_{i}, \mathbf{x}_{j}, t\right) V_{j}}{\sum_{j=1}^{N_{i}} V_{j}}
$$

Explicit time integration is used to find the temperature, velocity and displacement profile at each time step [43, 49]. The numerical procedure is provided in Fig. 2. In Fig. 2, $N_{t}$ represents the total number of time step, $n$ represents the current number of the time step, and $N_{\text {node }}$ represents the total number of PD points.

\section{Numerical results for verification problems}

In this section, peridynamic simulations are conducted by using the proposed model. The validity of the fully coupled thermomechanical equations is established by comparing the PD solutions with previously considered BEM and ANSYS solutions. Firstly, a dimensionless isotropic plate is imposed with three types of loadings, i.e. pressure shock loading, thermal shock loading and their combination. The results from PD solution are in agreement with the ones from an existing BEM solution. As a next verification problem, a block is investigated with a temperature boundary condition. Good agreements are obtained by comparing with PD and ANSYS solutions. Hence, the non-dimensional form of the equations is validated for both twoand three-dimensional problems. Secondly, a dimensional isotropic square plate is separately subjected to a tension pressure shock loading and a combination of compression and tension pressure shock loading. The temperature and displacement responses from PD solution coincide very well with ANSYS solution. Thus, present PD model is thus validated via these numerical simulations.

Subsequently, proposed model is further employed for failure prediction including fully coupled analysis. A plate with a pre-existing crack is studied under a pressure shock-loading condition. The temperature and structural responses without crack propagation are verified against ANSYS solution. Then, the crack propagation is simulated and its path is compared with the one from pure mechanical simulation. In this way, the coupling term effect on crack propagation is estimated and analysed.

\subsection{Plate subjected to shock loading}

The validity of the non-dimensional thermoelastic PD equations for 2D problems is established by constructing PD solutions for an existing BEM solution provided by Hosseini-Tehrani and Eslami [54]. The same geometry model, boundary conditions and loading conditions are adopted as in [54]. As shown in Fig. 3, a thin plate with a non-dimensional geometry $\bar{L}=10, \bar{W}=10$ and thickness $\bar{h}=1$ is subject to a shock loading on the edge of $\bar{x}=-\bar{L} / 2$ and fixed on the edge of $\bar{x}=\bar{L} / 2$. The edges of $\bar{y}=\bar{W} / 2$ and $\bar{y}=-\bar{W} / 2$ are traction free. Furthermore, at $\bar{x}=-\bar{L} / 2$ the plate is subjected to temperature boundary condition and all other three edges are insulated. The Poisson's ratio is set to be 0.17 . Regarding the PD discretization, the grid size is $\Delta x=0.05$ and the horizon size is chosen as $\delta=3.015 \Delta x$. The uniform time step size is $5.0 \times 10^{-4}$ with total simulation time $\bar{t}_{\text {total }}=6$. The boundary condition is implemented by using fictitious layers $[43,44]$. The applied loads are implemented on real boundary layer [49]. Since there is no heat source in this simulation case, $\bar{h}_{s}=0$.

The initial conditions are:

$$
\begin{aligned}
\bar{u}_{\bar{x}}(\bar{x}, \bar{y}, \bar{t}=0) & =\bar{u}_{\bar{y}}(\bar{x}, \bar{y}, \bar{t}=0)=0 \\
\bar{T}(\bar{x}, \bar{y}, \bar{t}=0) & =0
\end{aligned}
$$

with $\bar{u}_{\bar{x}}$ and $\bar{u}_{\bar{y}}$ representing the non-dimensional displacement components in the $x$ and $y$ directions, respectively.

The shock-loading conditions are: 


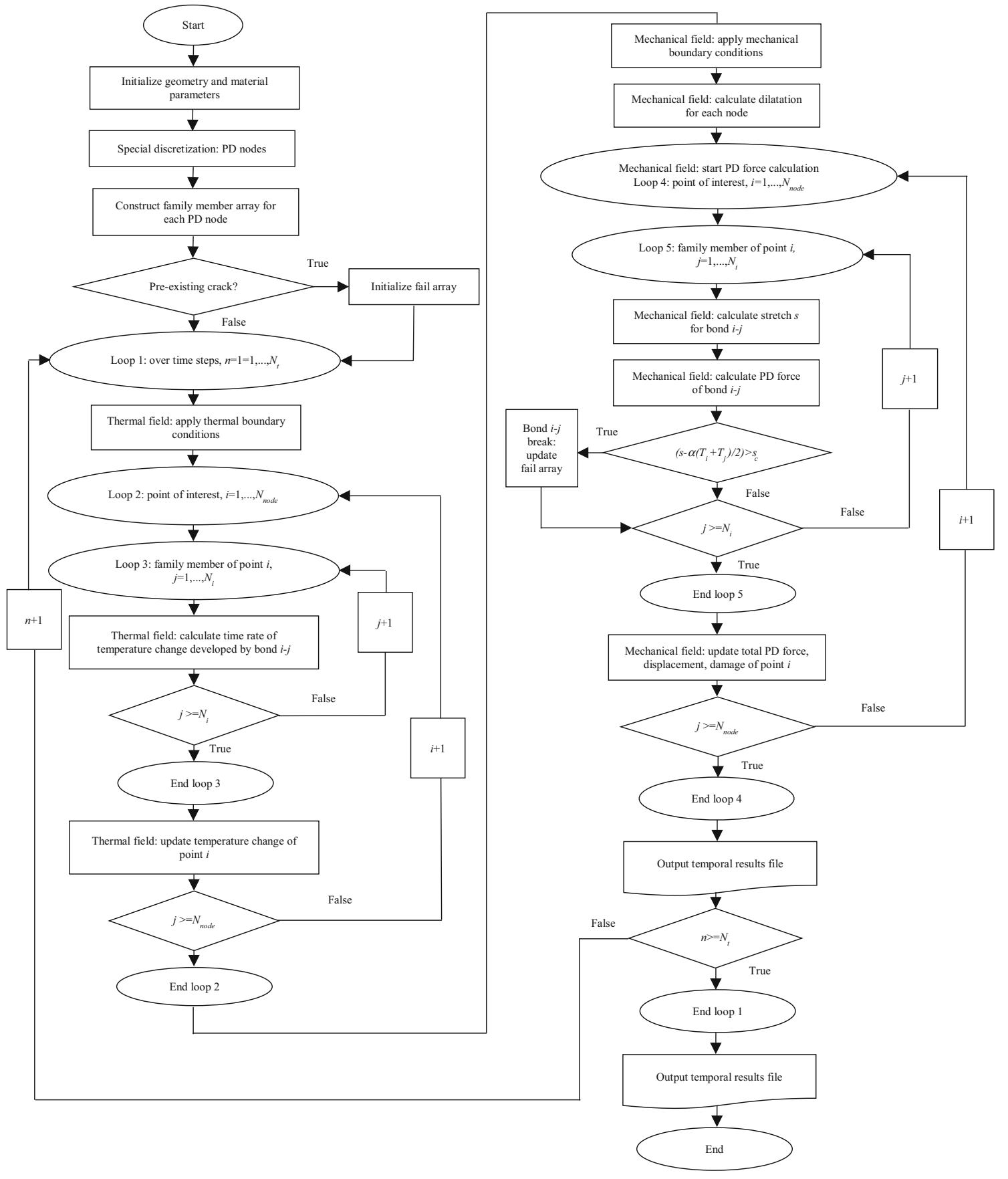

Fig. 2 Flow chart for the numerical procedure

Loading 1: Pressure shock

$$
\begin{aligned}
\bar{P}(\bar{x} & =-\bar{L} / 2, \bar{y}, \bar{t})=5 \bar{t} e^{-2 \bar{t}} \\
\bar{T}(\bar{x}=-\bar{L} / 2, \bar{y}, \bar{t}) & =0
\end{aligned}
$$

Loading 2: Thermal shock

$$
\begin{aligned}
& \bar{P}(\bar{x}=-\bar{L} / 2, \bar{y}, \bar{t})=0 \\
& \bar{T}(\bar{x}=-\bar{L} / 2, \bar{y}, \bar{t})=5 \bar{t} e^{-2 \bar{t}}
\end{aligned}
$$




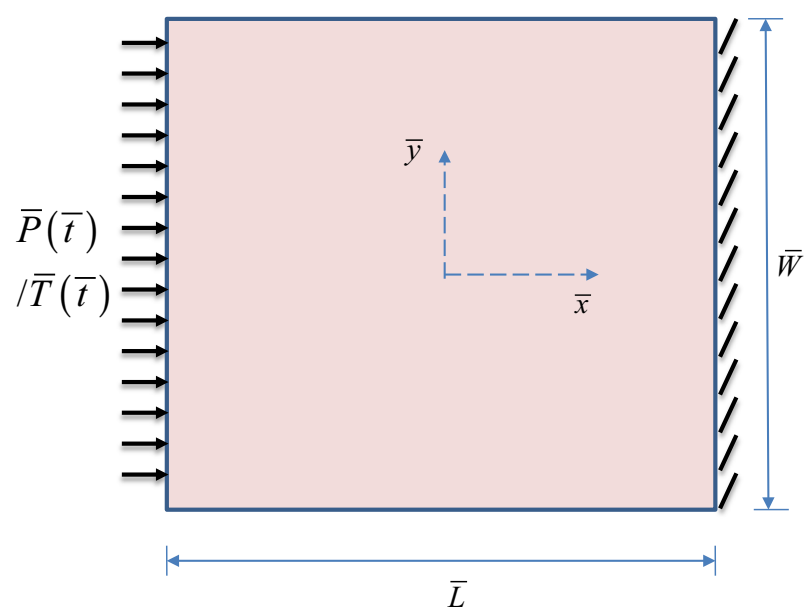

Fig. 3 A thin plate subjected to shock-loading conditions

Loading 3: Combined pressure and thermal shock

$$
\begin{aligned}
\bar{P}(\bar{x} & =-\bar{L} / 2, \bar{y}, \bar{t})=5 \bar{t} e^{-2 \bar{t}} \\
\bar{T}(\bar{x}=-\bar{L} / 2, \bar{y}, \bar{t}) & =5 \bar{t} e^{-2 \bar{t}}
\end{aligned}
$$

where $\bar{t}$ represents the non-dimensional time. The applied pressure load is in the positive $x$ direction, as illustrated in Fig. 3.

Figures 4, 5 and 6 provide the thermal and mechanical responses at $\bar{t}=3$ and $\bar{t}=6$ along the horizontal centreline of the plate for 3 different loading conditions. The coupling coefficient $\epsilon=0$ represents the uncoupled case, where the effect of deformation on temperature field is ignored. Figure $4 \mathrm{a}$ represents the temperature distribution when the plate is subjected to pure pressure shock loading (loading condition 1). As it can be seen from the figure, when $\epsilon=0$ no temperature change is observed. On the other hand, when $\epsilon=0.1$ both temperature drop and temperature rise are observed, which are induced by the applied pressure shock due to coupling effect. The magnitude of the temperature change is relatively small, within the range between -0.02 and 0.05 . As the time progresses, the peak position of the temperature distribution moves towards the positive $x$ direction. Figure $4 \mathrm{~b}$ represents the dimensionless axial displacement along the horizontal centreline at $\bar{t}=3$ and $\bar{t}=6$. The wave fronts at these two time points are clearly observed. There are slight differences between the displacement predictions from the coupled and uncoupled cases. As the time progresses, the difference becomes larger. Therefore, it could be inferred that due to coupling effect, the temperature change induced by deformation does affect the deformation. The same conclusion is obtained from the simulation cases with loading condition 2 as can be seen from Fig. 5. Due to the heating effect by the applied thermal loading, the plate experiences an expansion state. Subsequently, the tension loading creates a cooling effect. Therefore, when compared with the uncoupled case, relatively lower temperature change is observed in coupled case. Consequently, the lower temperature change gives rise to a smaller deformation response. This conclusion can also be applied loading condition 3, which results are presented in Fig. 6.

In conclusion, good agreements are obtained for three types of loadings. For both the thermal and mechanical fields, the results from ordinary state-based PD predictions agree well with those from BEM solutions obtained by Hosseini-Tehrani and Eslami [54]. Therefore, via these numerical simulations, the present nondimensional PD model is validated for 2D problems.

\subsection{Plate subjected to pressure loading}

Thermal and deformation responses of a square plate under two types of pressure shocks are analysed. The geometry dimensions of the plate are $L=W=0.1 \mathrm{~m}$, with the thickness being $0.001 \mathrm{~m}$. The material is chosen as carbon steel, and its material properties are given as: elastic modulus $E=200 \mathrm{GPa}$, Poisson's ratio $v=0.17$, thermal expansion coefficient $\alpha=11.5 \times 10^{-6} \mathrm{~K}^{-1}$, density $\rho=7870 \mathrm{~kg} / \mathrm{m}^{3}$, specific heat capacity $c_{\mathrm{V}}=472 \mathrm{~J} /(\mathrm{kgK})$ and thermal conductivity $k_{\mathrm{T}}=51.9 \mathrm{~W} /(\mathrm{mK})$. The reference temperature is 


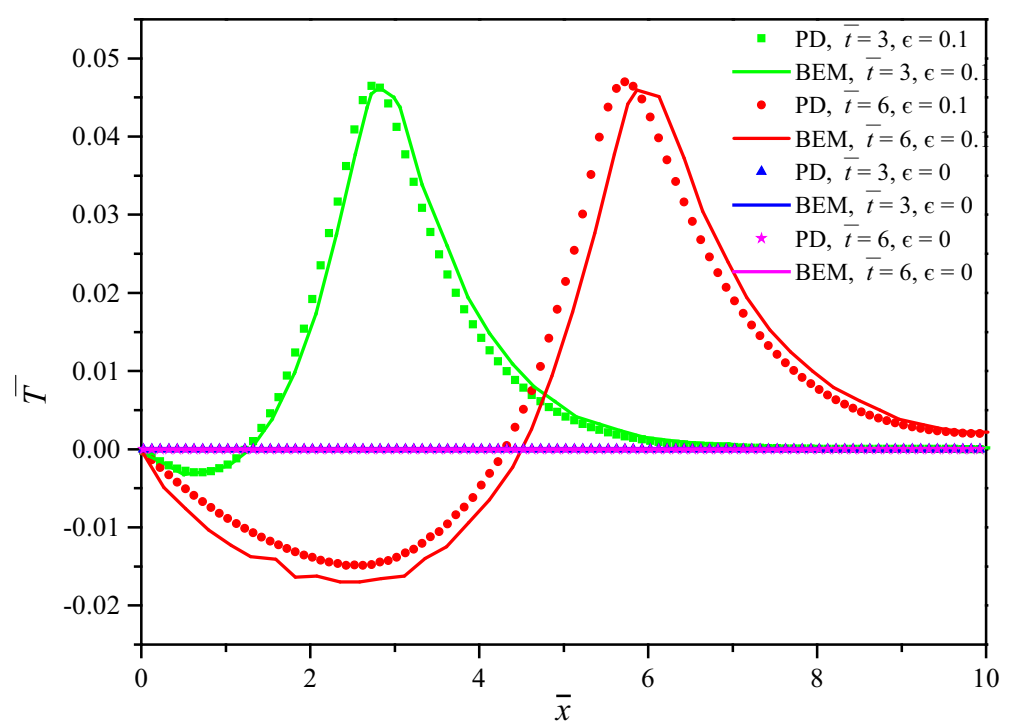

(a)

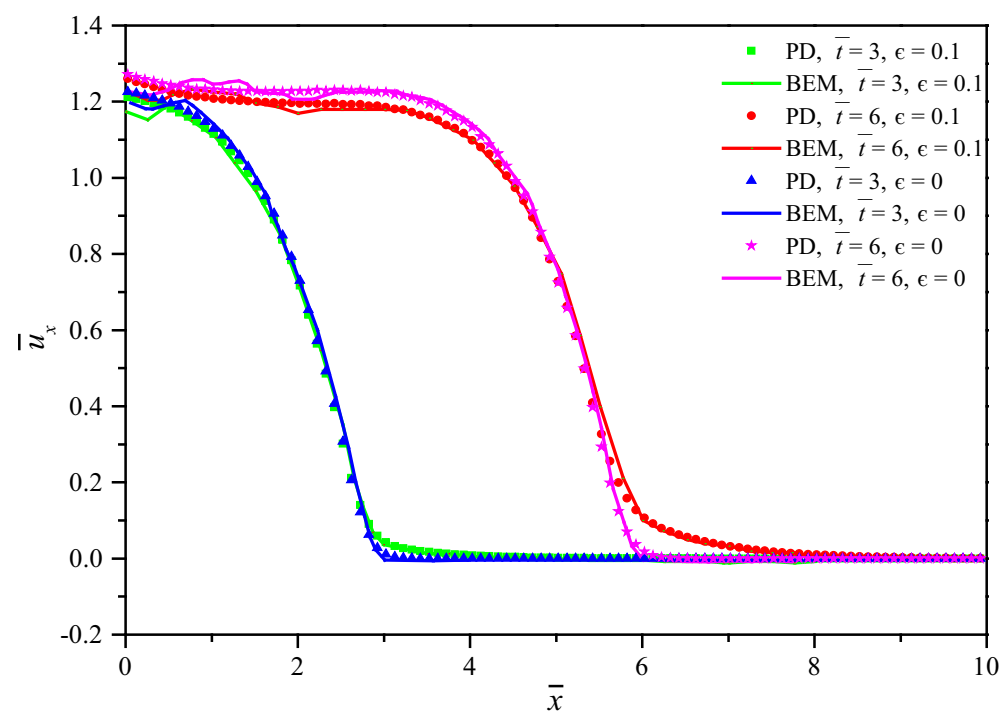

(b)

Fig. 4 Comparison of BEM method [54] and PD solutions a temperature change distributions, b displacements along the horizontal centreline of the plate for loading condition 1

$\Theta_{0}=285 \mathrm{~K}$. The grid size is $\Delta x=0.0005 \mathrm{~m}$, and the horizon size is chosen as $\delta=3.015 \Delta x$. The time step size is $0.001 \mu \mathrm{s}$ with total simulation time $8 \mu \mathrm{s}$. On the other hand, directly coupled plane element PLANE 223 is utilized in the ANSYS model. The number of nodes $80 \times 80$ and time step size is $0.16 \mu$ s for the ANSYS model.

The initial conditions are:

$$
\begin{array}{r}
u_{x}(x, y, t=0)=u_{y}(x, y, t=0)=0 \\
T(x, y, t=0)=0
\end{array}
$$

The shock-loading conditions are:

Loading 1:

$$
P(x=-L / 2, y, t)=-10^{20} t^{2} \mathrm{~Pa}
$$




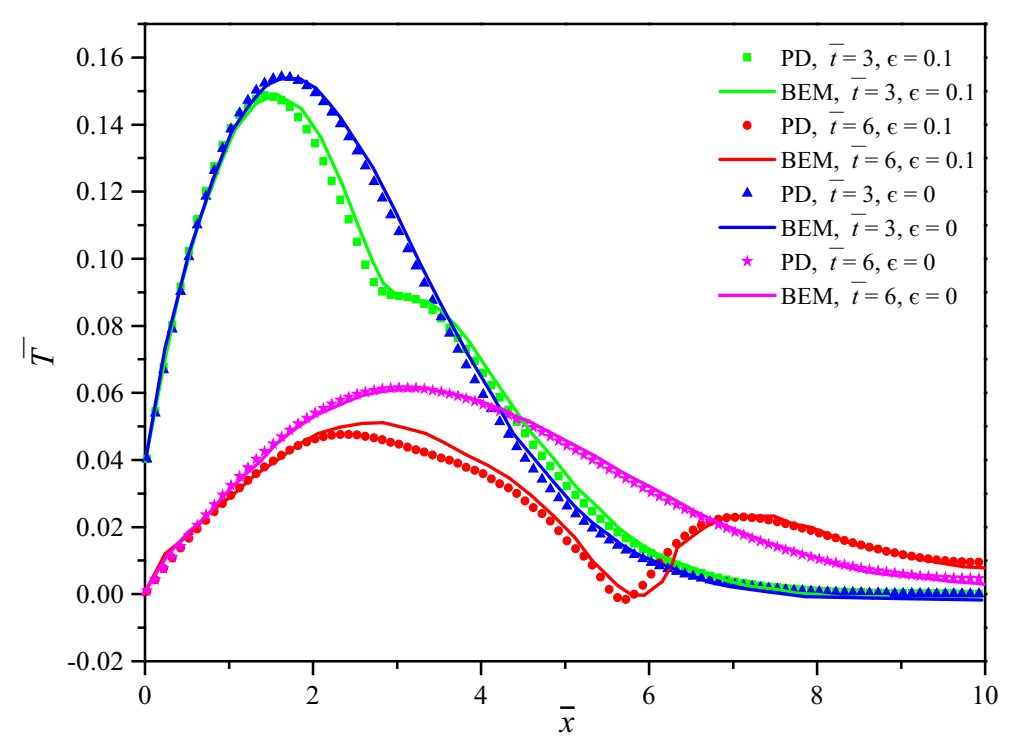

(a)

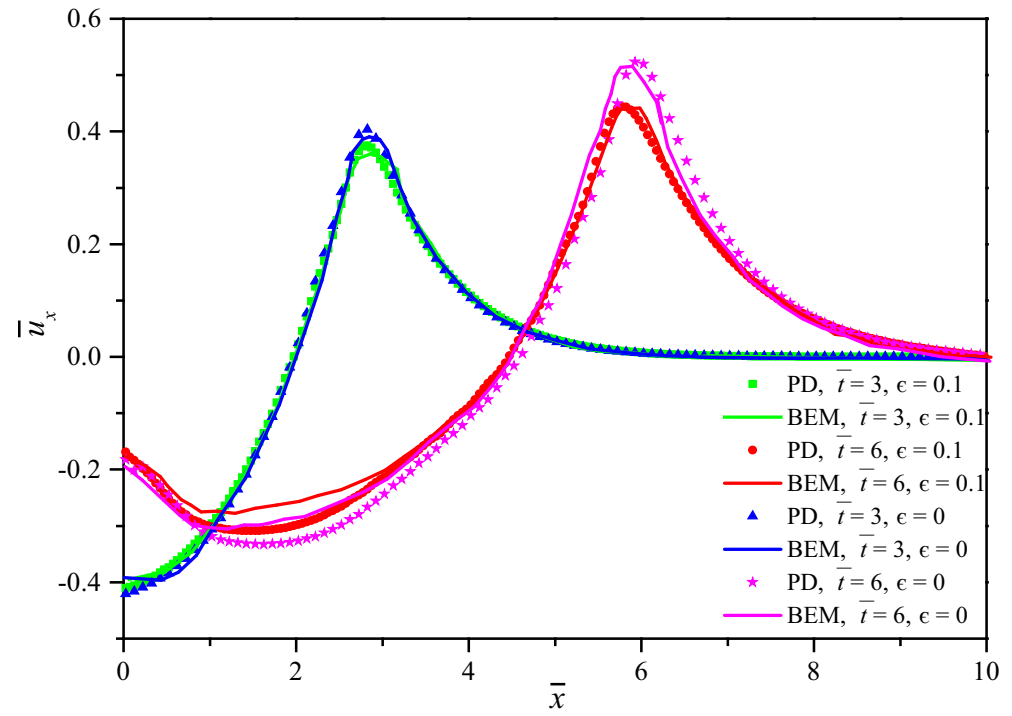

(b)

Fig. 5 Comparison of BEM method [54] and PD solutions a temperature change distributions, b displacements along the horizontal centreline of the plate for loading condition 2

Loading 2:

$$
P(x=-L / 2, y, t)=10^{9} \sin \left(\frac{\pi}{2} t \times 10^{6}\right) \mathrm{Pa}
$$

Figures 7 and 8 provide the temperature and deformation responses along the horizontal centreline of the plate under two loading conditions. Since only the mechanical loadings are applied, the temperature changes are induced due to coupling term in the heat flow equation. As shown in Figs. 7a and 8a, there is a remarkable agreement between PD and ANSYS solutions. When the plate is subjected to loading condition 1, temperature drop is observed due to tension loading as it can be seen from Fig. 7a. As the time progresses, temperature change increases to a final value of $6.5 \mathrm{~K}$. When the plate is subjected to loading condition 2, temperature increases where there is local compression and temperature drops where there is local tension.

Under both loading conditions displacement fields obtained from PD and ANSYS simulations match very well. Furthermore, it should be noted that even though the carbon steel has a relatively small coupling coefficient, i.e. $\epsilon=0.002861$, the generated temperature change due to mechanical shock loading is considerable. 


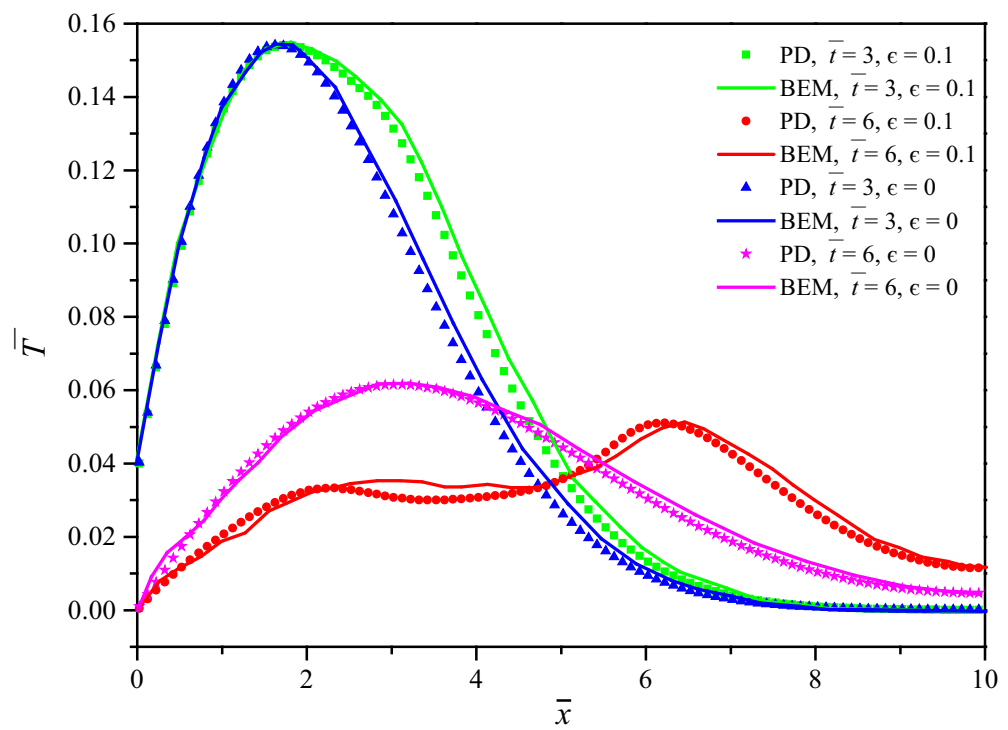

(a)

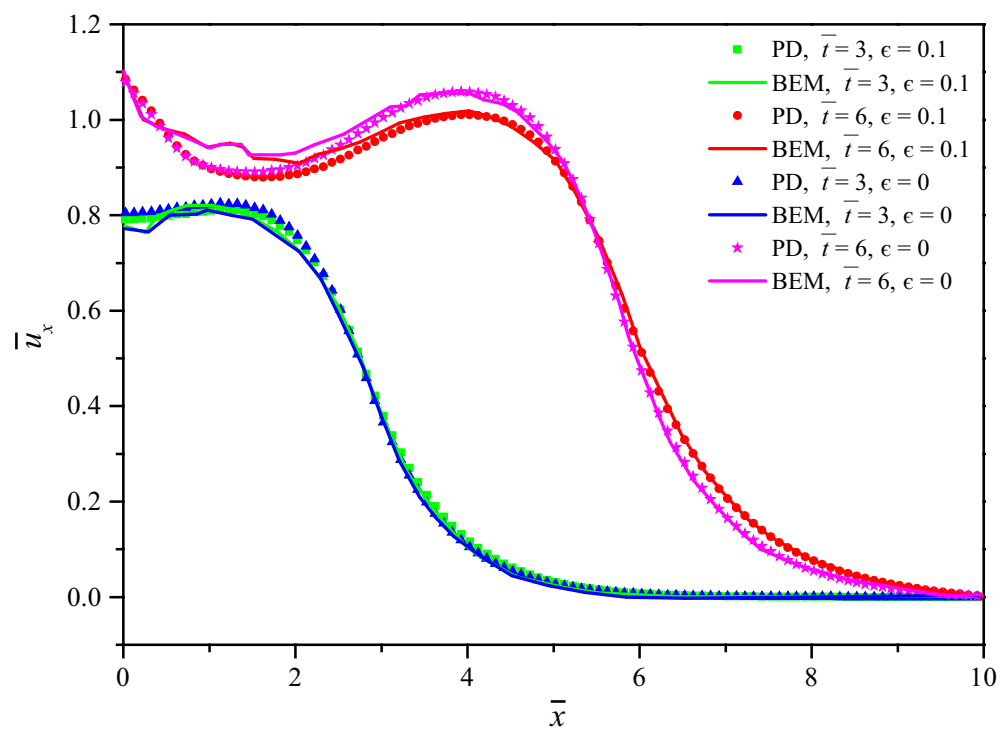

(b)

Fig. 6 Comparison of BEM method [54] and PD solutions a Temperature change distributions, b displacements along the horizontal centreline of the plate for loading condition 3

Therefore, if large strain rate exists, i.e. shock loading is applied, fully coupled thermomechanical analysis should be taken into consideration.

\subsection{Block subjected to thermal loading}

In order to validate the proposed PD model for 3D problems, a block subjected to temperature boundary condition is investigated. As shown in Fig. 9, the dimensionless length, width and height of the block are 5, 0.15 and 0.15 , respectively. The Poisson's ratio is set as 0.33 , and the coupling coefficient is 1.0. Regarding the PD model, the grid size is $\Delta x=0.0125$ and the horizon is chosen as $\delta=3.015 \Delta x$. The integration time step size is $\pi / 4 \times 10^{-4}$, and the total simulation time is $\pi$. On the other hand, directly coupled solid element-type SOLID 226 is adopted with mesh size of 0.05 and time step size of $0.02 \pi$ in ANSYS model. 


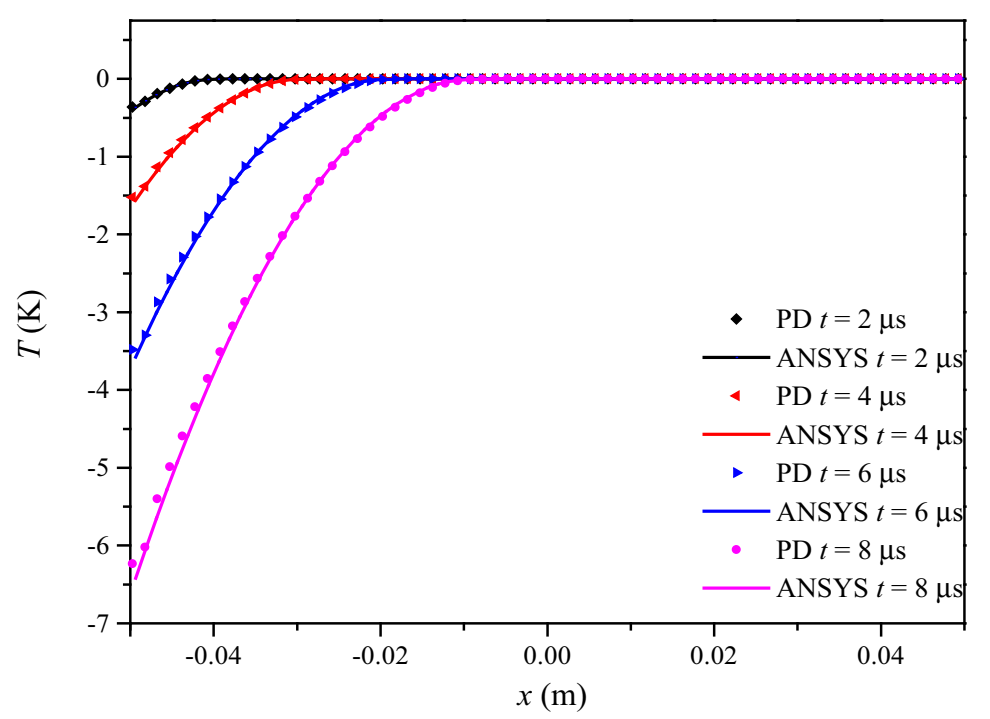

(a)

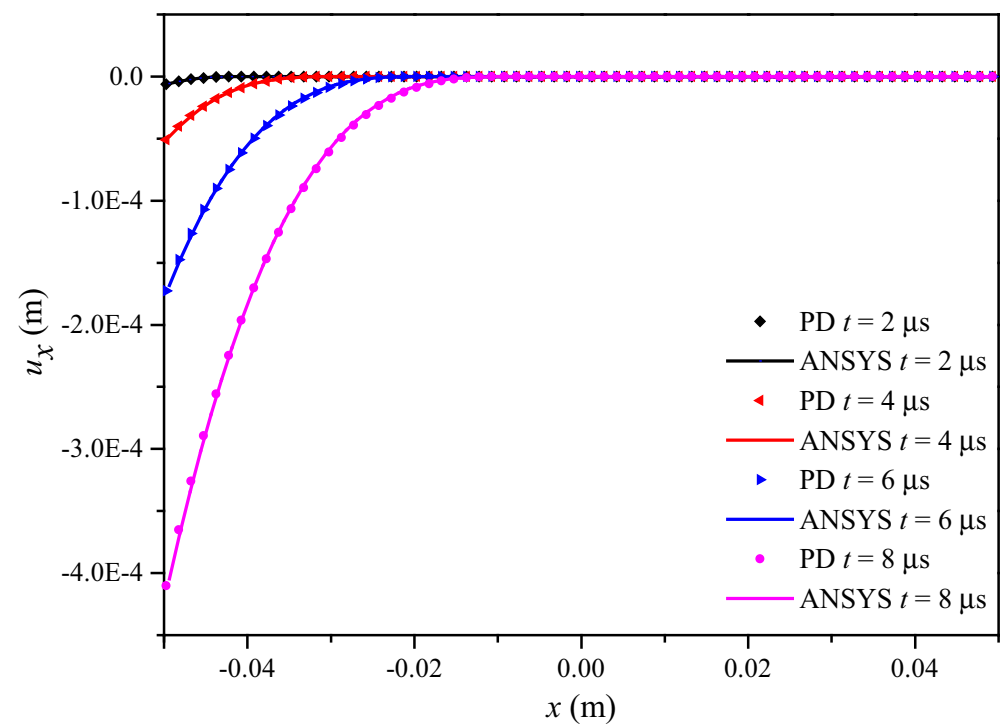

(b)

Fig. 7 Comparison of ANSYS and PD solutions a temperature change distributions, b displacements along the horizontal centreline of the plate for loading condition 1

The block is clamped at $x=\bar{L}$. The block is gradually heated at $x=0$, and all other surfaces are insulated. The temperature boundary condition is defined as $\bar{T}=\sin (\bar{t})$, where $\bar{t}$ is the dimensionless time. Fictitious layer is used to implement the boundary conditions [43].

The temperature distributions and horizontal displacements along the line of $y=\bar{W} / 2$ and $z=\bar{H} / 2$ are presented in Fig. 10a and $\mathrm{b}$ at dimensionless time of $\bar{t}=\pi / 4, \pi / 2,3 \pi / 4, \pi$, respectively. The results which are obtained from ANSYS solutions are also provided for comparison. It could be seen that both the temperature and displacement distributions match very well, indicating the capability of the derived PD formulations to accurately predict the thermal and mechanical responses for three-dimensional problems.

Derived formulations and explicit expressions of PD parameters including their dimensional and nondimensional forms are validated through these numerical simulations. 


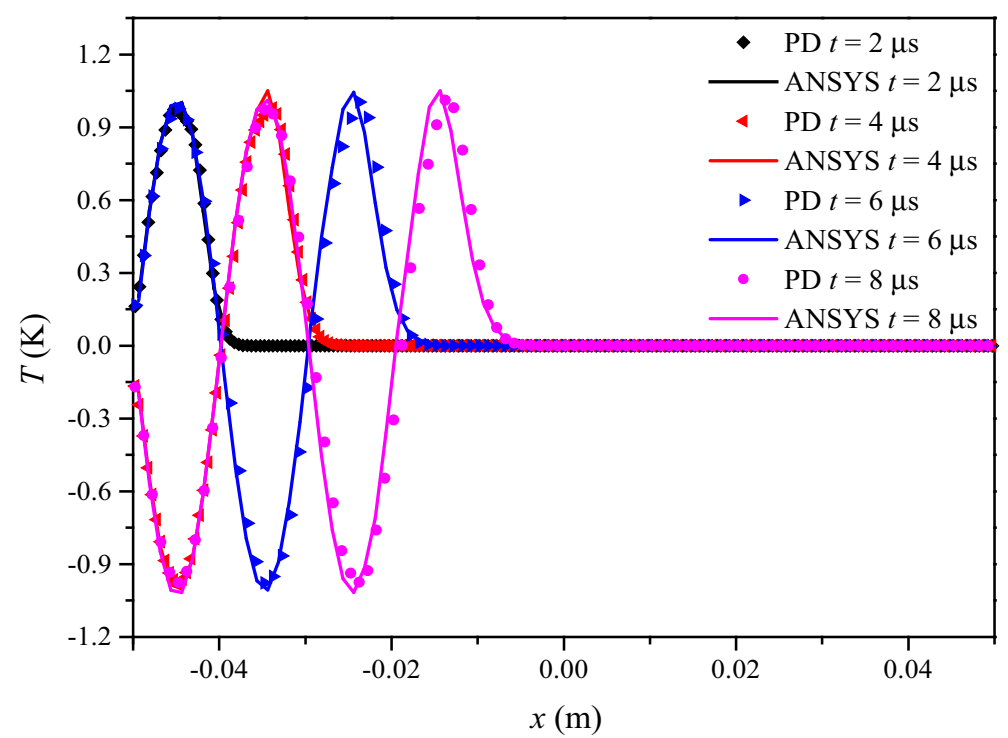

(a)

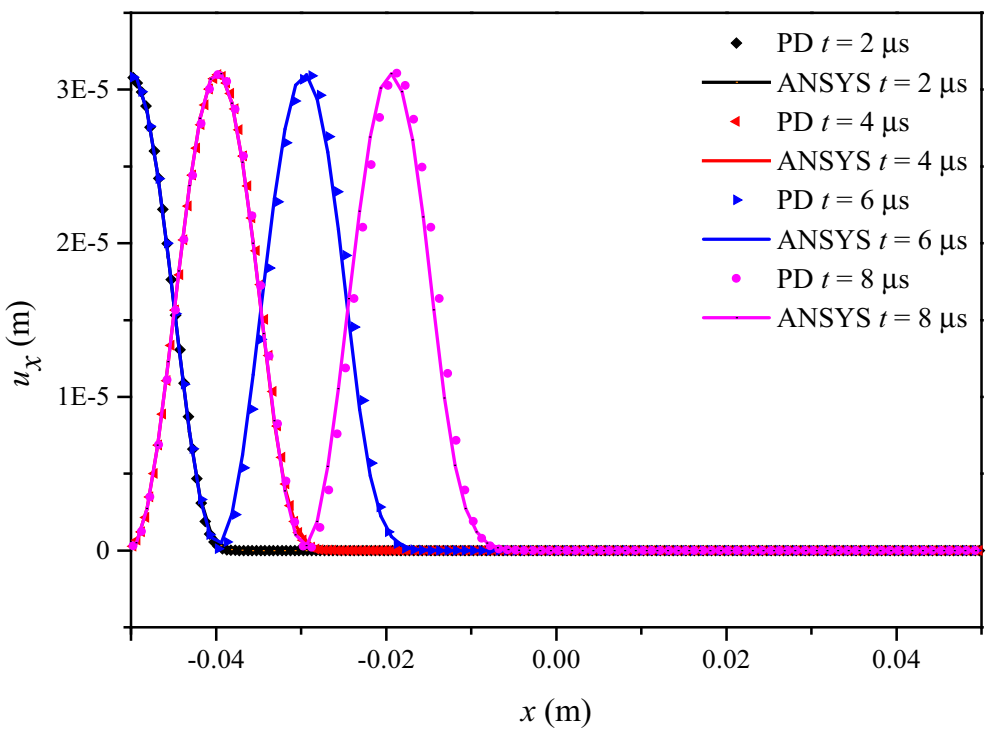

(b)

Fig. 8 Comparison of ANSYS and PD solutions a temperature change distributions, $\mathbf{b}$ displacements along the horizontal centreline of the plate for loading condition 2

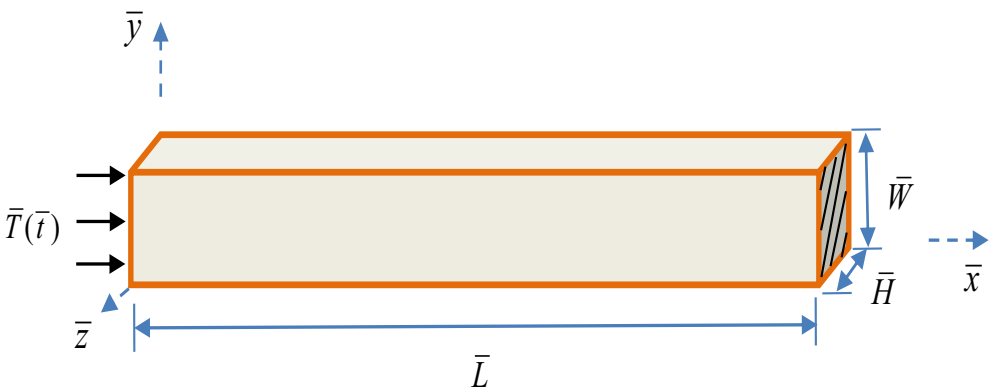

Fig. 9 Block under thermal loading 


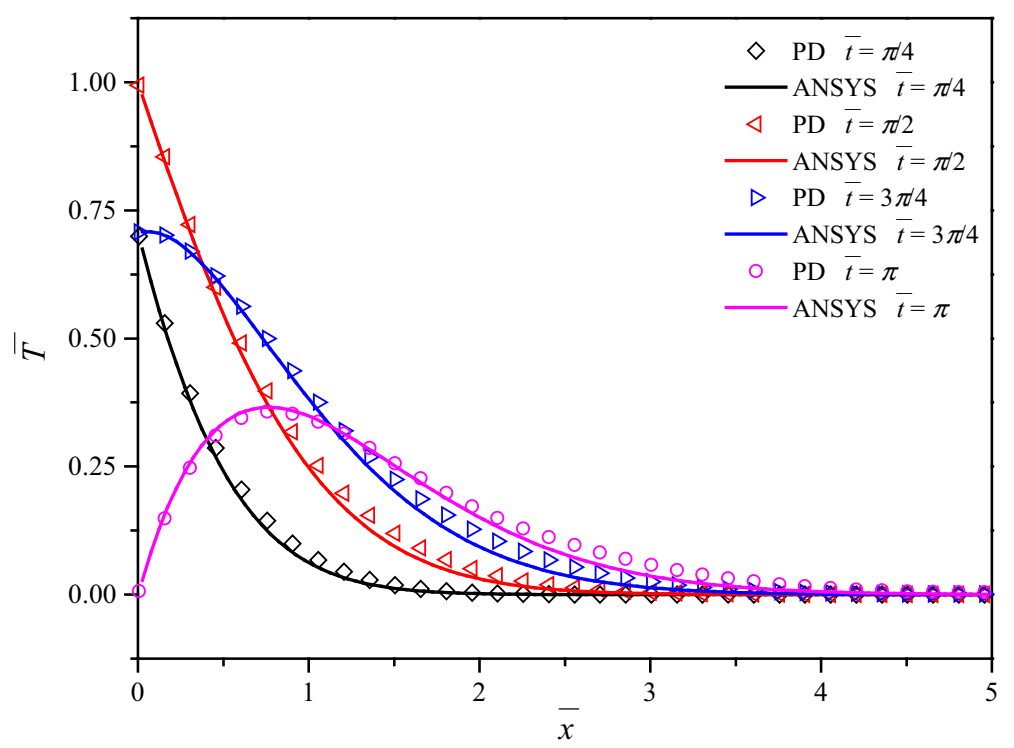

(a)

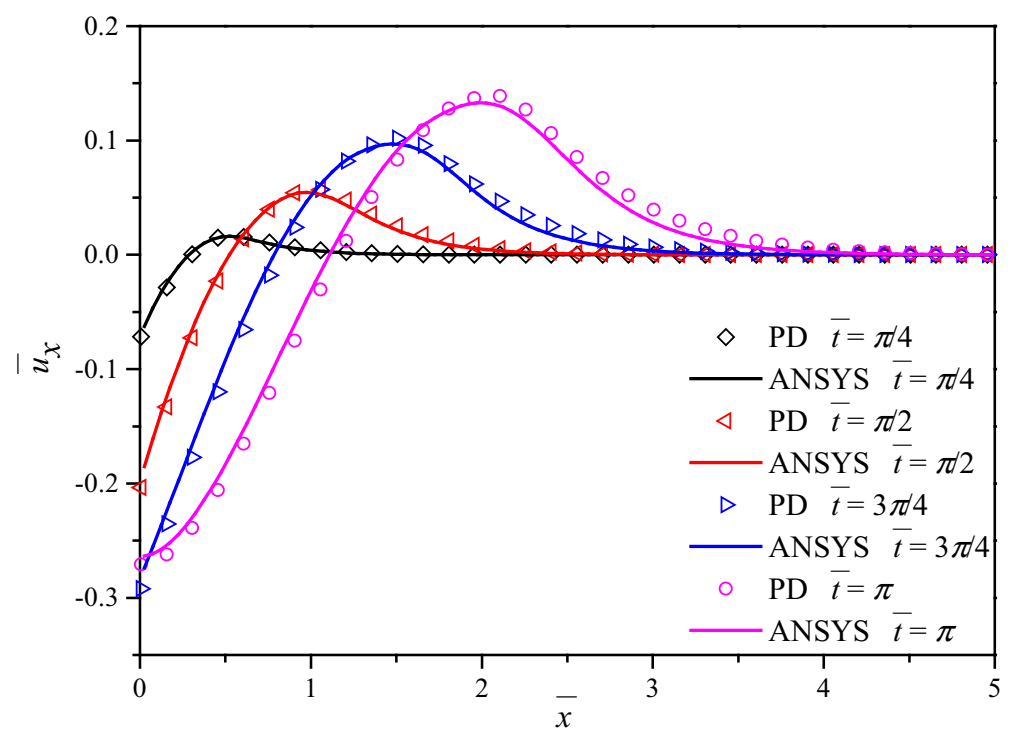

(b)

Fig. 10 Comparison of ANSYS and PD solutions a temperature change distributions, b horizontal displacements along the centreline of the block

\section{Numerical results for damage prediction}

Peridynamics is a reformulation of classical equations that is better suited for modelling bodies with discontinuities. Therefore, analyses involving cracks are considered in this study by using the developed PD model. First, a three-point bending test is considered to predict the temperature distribution and deformed shape. Then a plate with a pre-existing central crack subjected to a pressure shock-loading condition is investigated. The temperature distributions and crack propagation paths are investigated. In addition, the crack propagation paths without considering coupling terms are also predicted for comparison. Finally, a numerical simulation based on Kalthoff experiment [55] is carried out. The crack path predicted by PD model is compared with the result of the experiment. 


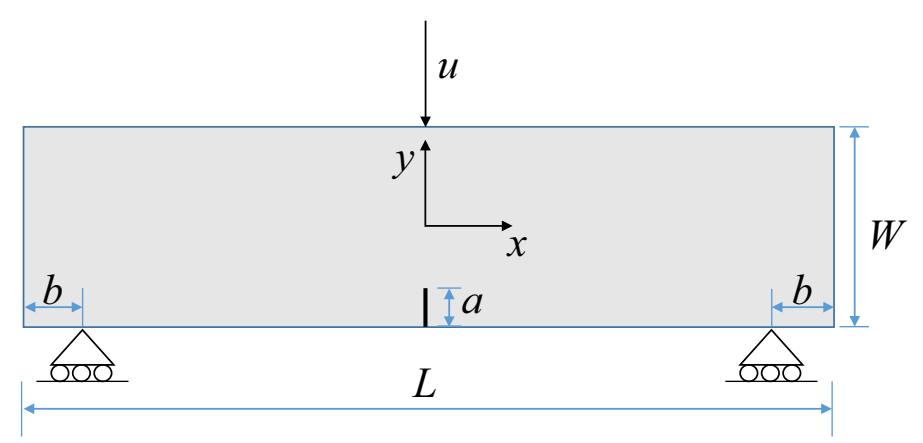

Fig. 11 Geometry of three-point bending test

\subsection{Three-point bending simulation}

Three-point bending test of a simply supported beam is simulated under prescribed displacement condition as illustrated in Fig. 11. The origin of the coordinate system is located in the middle of the beam. The material properties are related to rubber like material, shear modulus is $1 \mathrm{MPa}$, Poisson's ratio $v=0.45$, thermal expansion coefficient $\alpha=1 \times 10^{-3} \mathrm{~K}^{-1}$, thermal conductivity $k_{\mathrm{T}}=0.1 \mathrm{~W} /(\mathrm{mK})$, density $\rho=906.5 \mathrm{~kg} / \mathrm{m}^{3}$, specific heat capacity $c_{\mathrm{v}}=1103.14 \mathrm{~J} /(\mathrm{kgK})$ [56]. The critical stretch value is $s_{\mathrm{c}}=0.1724$. The reference temperature is $\Theta_{0}=293 \mathrm{~K}$. The dimensions of the beam are $L=40 \mathrm{~mm}, W=10 \mathrm{~mm}$, and thickness $h=$ $0.5 \mathrm{~mm}$. A pre-existing crack with length being $a=1 \mathrm{~mm}$ is located in the middle of the beam at $y=-W / 2$. The supports are placed $b=4 \mathrm{~mm}$ inwards from the outer edges. A prescribed displacement applied in the middle of beam at $y=W / 2$. The prescribed displacement at $y=W / 2$ is set to be $u=\left(t / t_{\text {total }}\right) u_{\text {max }}$, where $t_{\text {total }}$ is the total simulation time, $t_{\text {total }}=0.01 \mathrm{~s}$ and $u_{\max }=6 \mathrm{~mm}$. The uniform PD grid size is $\Delta x=0.5 \mathrm{~mm}$, and the horizon is $\delta=3.015 \Delta x$. The time step size is $1 \times 10^{-6} \mathrm{~s}$, and the total simulation time is $t_{\text {total }}=0.01 \mathrm{~s}$.

Figures 12 and 13 present the damage plot and corresponding temperature distributions at $t=0.007 \mathrm{~s}$ and $t=0.01 \mathrm{~s}$. It can be observed from Figs. 12a and 13a that the initial crack grows in the vertical direction. Figures $12 \mathrm{~b}$ and $13 \mathrm{~b}$ present the corresponding temperature distributions. As expected, temperature rise is observed where local compression is expected and temperature drop is observed near the crack surfaces where there is local tension.

\subsection{Plate with a pre-existing central crack subjected to pressure shock loading}

Based on the previous example in Sect. 3.2, damage propagation is predicted for a plate with pre-existing crack of length $0.02 \mathrm{~m}$, as shown in Fig. 14. The geometry, material properties and boundary condition are identical to the those in Sect. 3.2.

The pressure shock loading is specified as

$$
P(t)=\left\{\begin{array}{l}
-1 \times 10^{14} t(\mathrm{~Pa}), t<t_{0} \\
-1 \times 10^{14} t_{0}(\mathrm{~Pa}), t>t_{0}
\end{array}\right.
$$

where $t_{0}=8 \mu \mathrm{s}$. The total node number in $x$ or $y$ direction for PD model is set to be 500 with a grid size of $0.2 \mathrm{~mm}$. Thus, the critical stretch value $s_{\mathrm{c}}$ is calculated as 0.0213 with $G_{\mathrm{c}}$ being $42320 \mathrm{~J} / \mathrm{m}^{2}$. The horizon is $\delta=3.015 \Delta x$. The time step size is set as $10^{-9} \mathrm{~s}$, and the total simulation time is $30 \mu \mathrm{s}$. On the other hand, directly coupled plane element-type PLANE223 is applied in the ANSYS model with the grid size of $\Delta x=0.00125$ and time step size of $0.6 \mu \mathrm{s}$.

In order to better understand the existence of crack surface on the temperature and deformation field, initially failure is not allowed. The horizontal displacement predictions at different time steps are shown in Figs. 15, 16 and 17. It is observed that the peridynamic results coincide very well with ANSYS solutions. The displacement distribution along $x$-axis propagates uniformly in the vertical direction before the elastic wave reaches the crack, as shown in Fig. 15. After the elastic wave reaches the crack surface, displacements become non-uniform due to the discontinuity of the displacements along the crack surface as it can be seen from Figs. 16 and 17. 


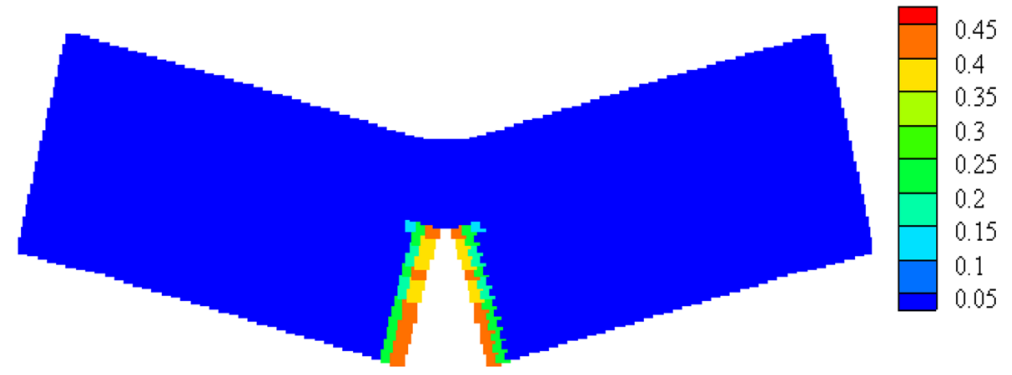

(a)

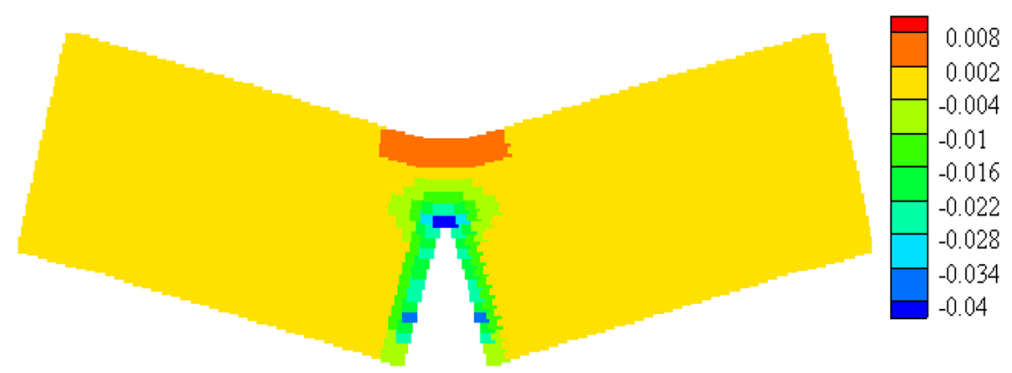

(b)

Fig. 12 a Damage plot and $\mathbf{b}$ temperature change $(\mathrm{K})$ in deformed configuration at $t=0.007 \mathrm{~s}$ (displacement scale factor is 1 )

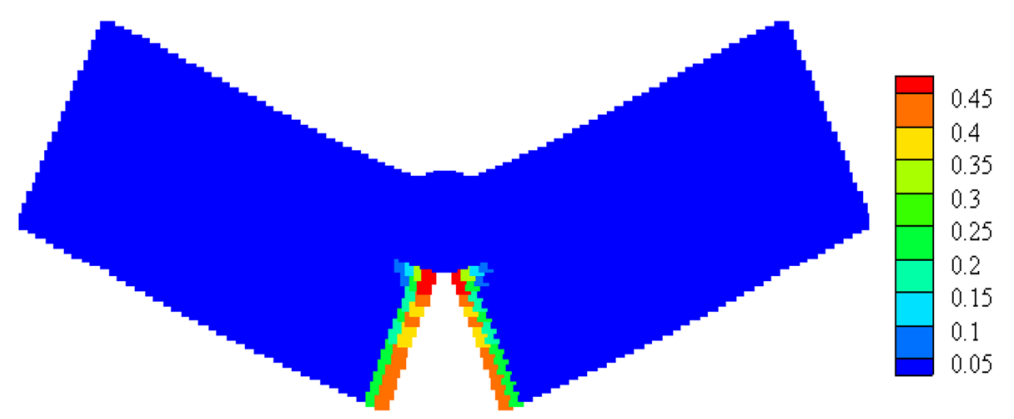

(a)

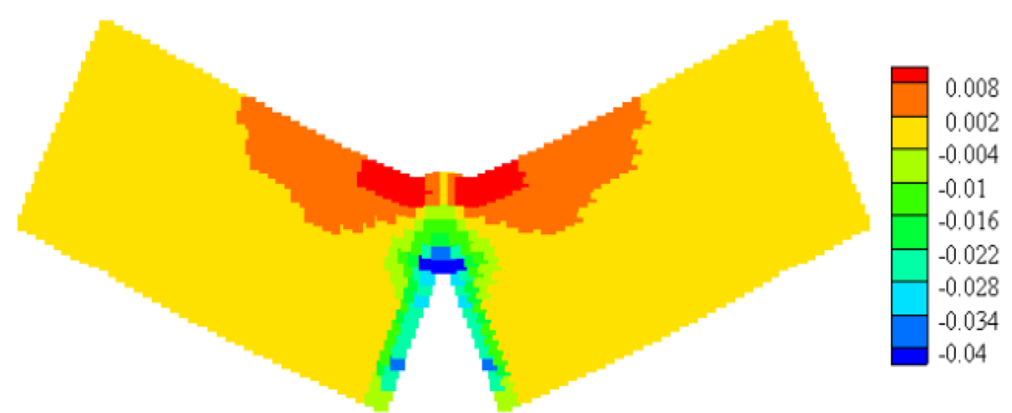

(b)

Fig. 13 a Damage plot and $\mathbf{b}$ temperature change $(\mathrm{K})$ in deformed configuration at $t=0.01 \mathrm{~s}$ (displacement scale factor is 1 ) 


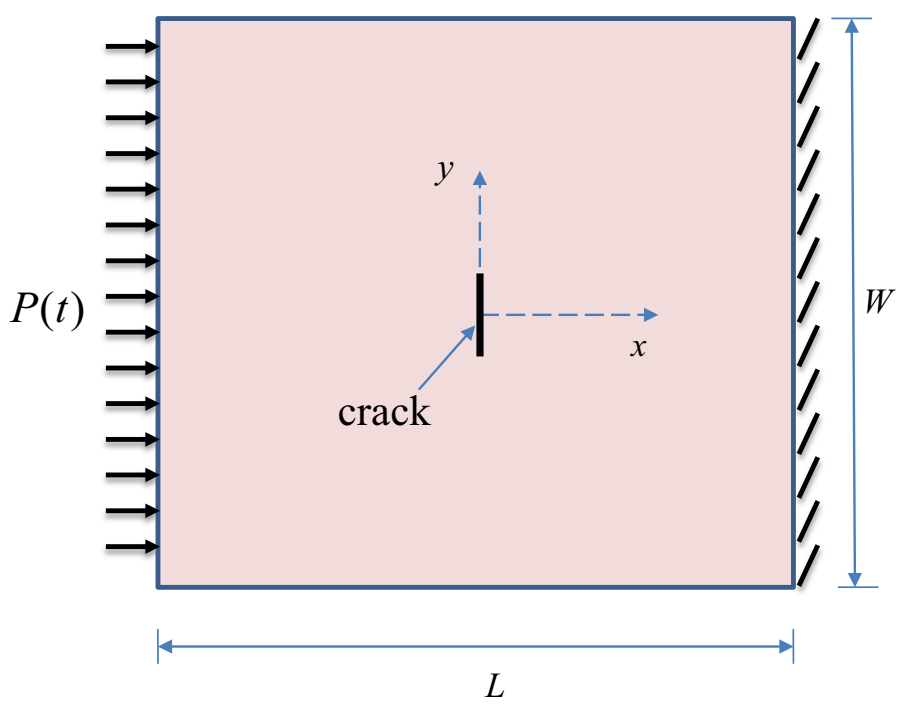

Fig. 14 An isotropic plate with a pre-existing central crack under pressure shock loading

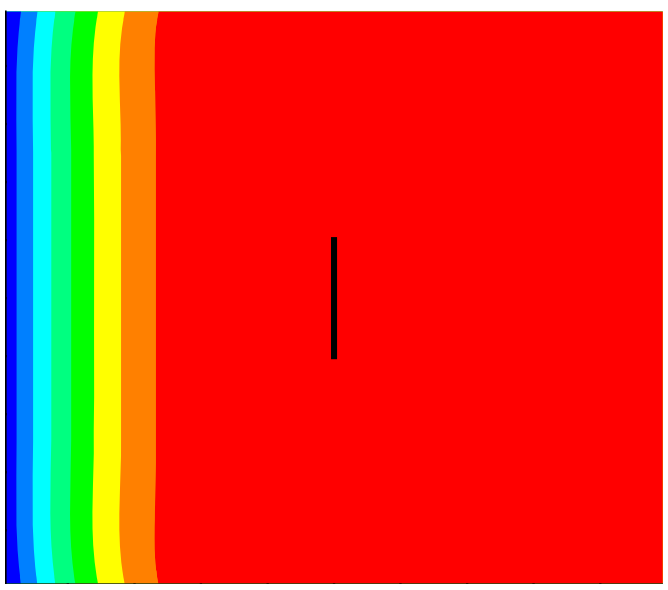

(a)

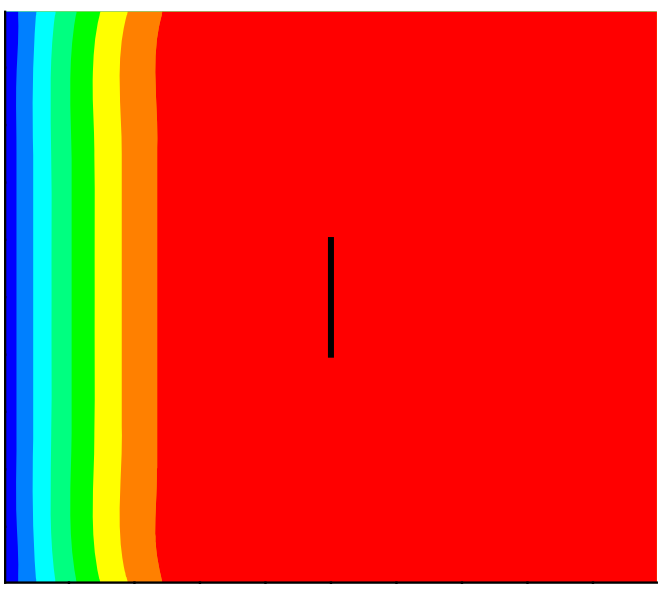

(b)

Fig. 15 Horizontal displacements (m) a ANSYS and b PD results at $t=7 \mu \mathrm{s}$

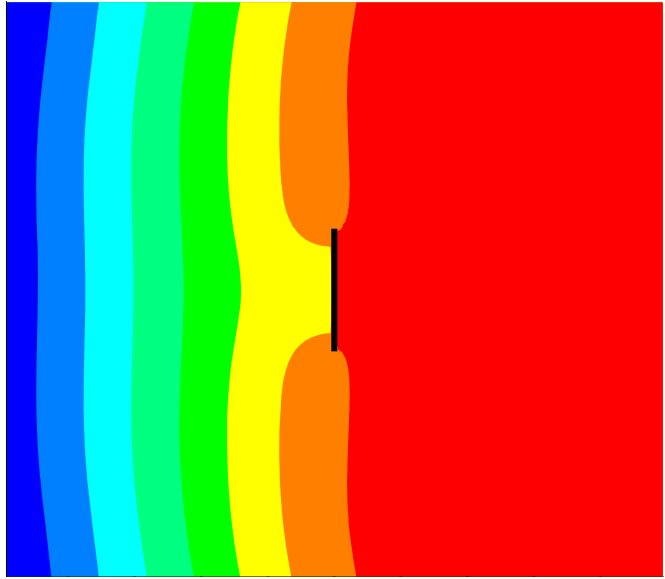

(a)

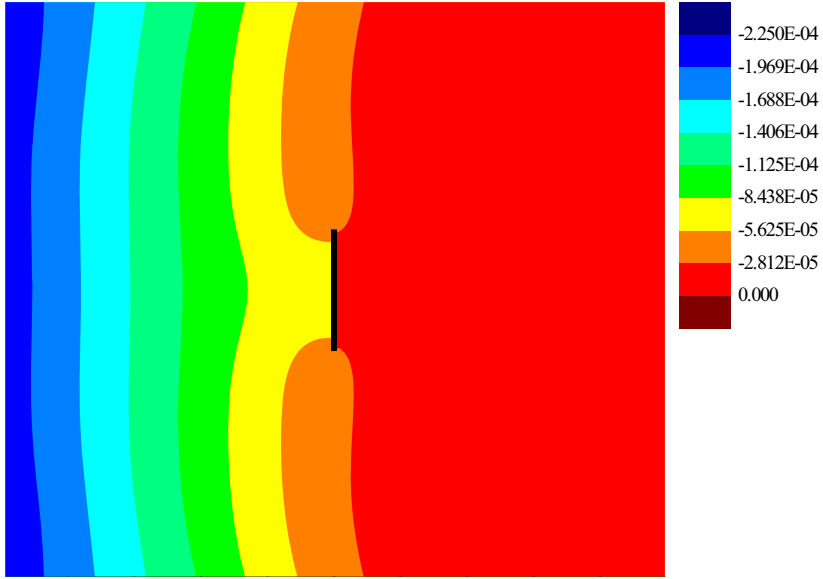

(b)

Fig. 16 Horizontal displacements (m) a ANSYS and $\mathbf{b}$ PD results at $t=15 \mu \mathrm{s}$ 


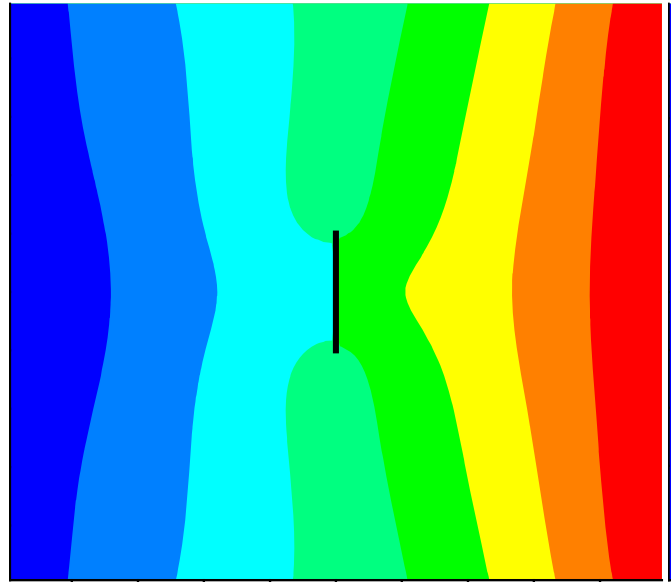

(a)

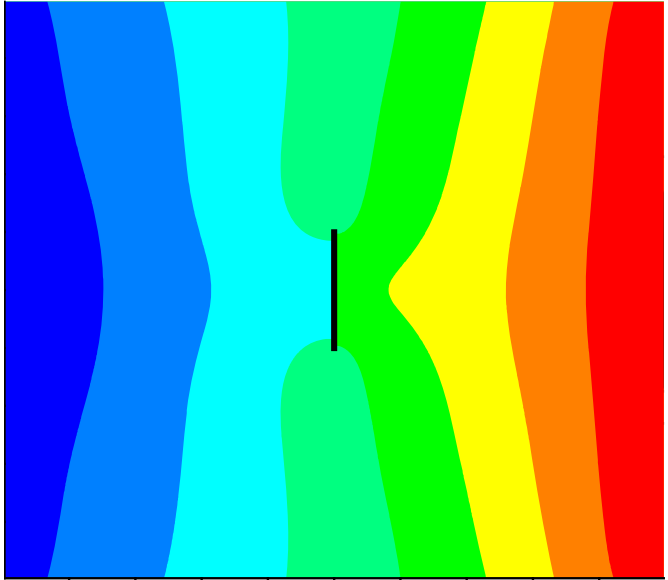

(b)

Fig. 17 Horizontal displacements (m) a ANSYS and $\mathbf{b}$ PD results at $t=30 \mu \mathrm{s}$

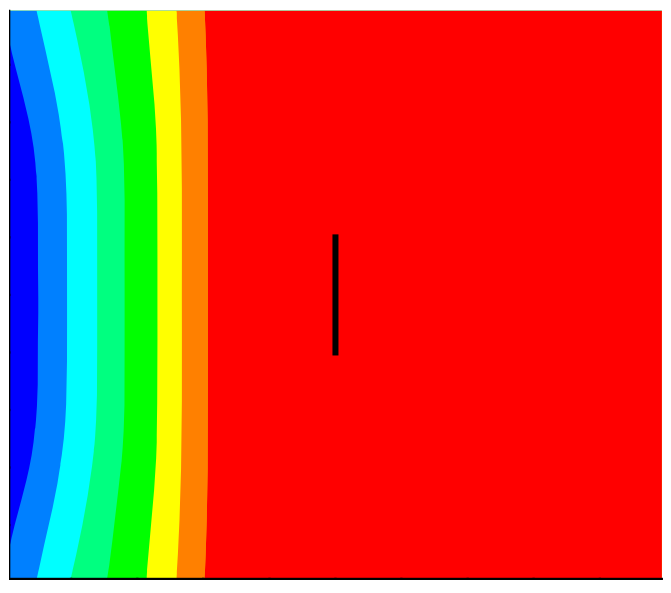

(a)

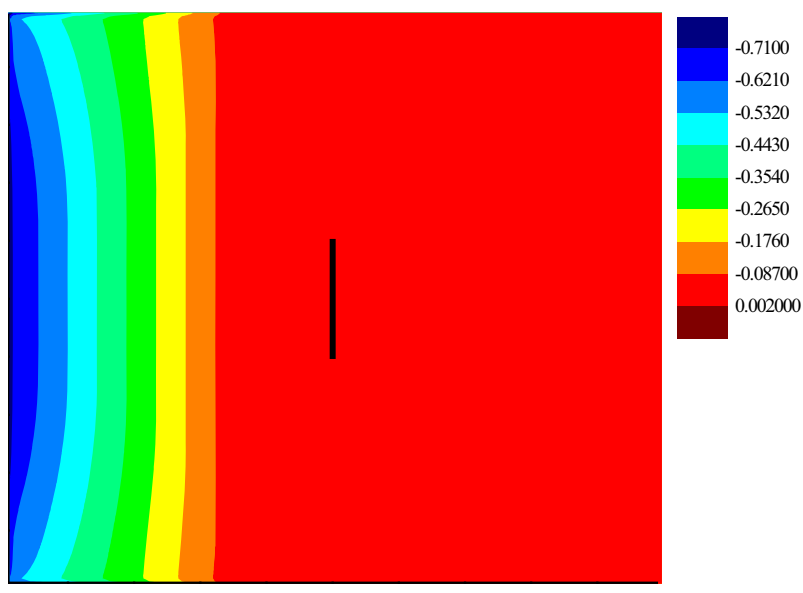

(b)

Fig. 18 Temperature change distributions (K) a ANSYS and $\mathbf{b}$ PD results at $t=7 \mu \mathrm{s}$

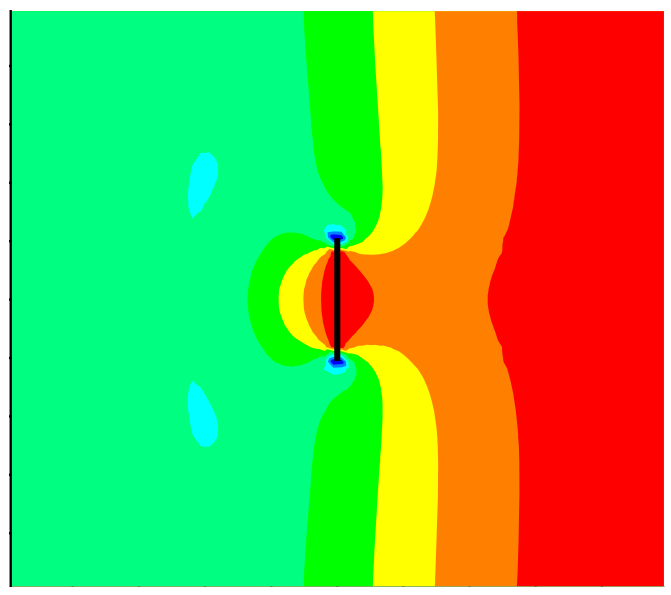

(a)

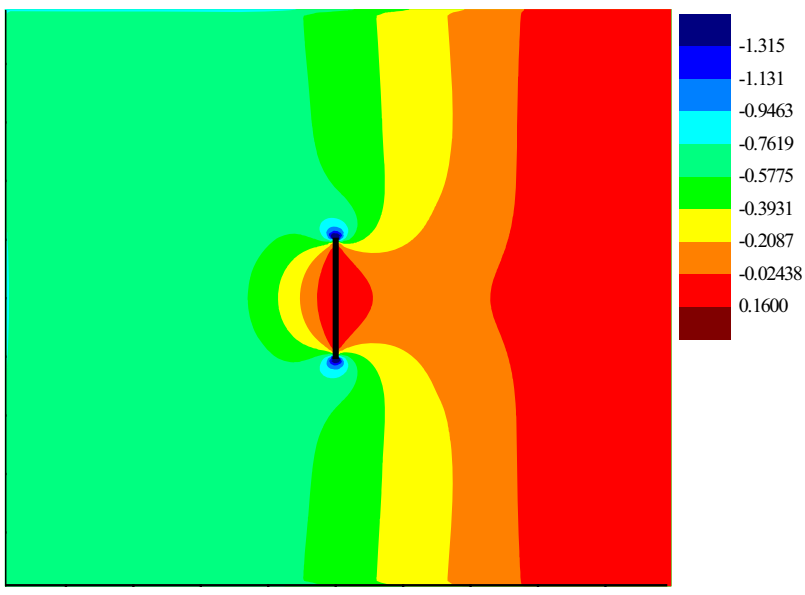

(b)

Fig. 19 Temperature change distributions (K) a ANSYS and b PD results at $t=15 \mu \mathrm{s}$ 


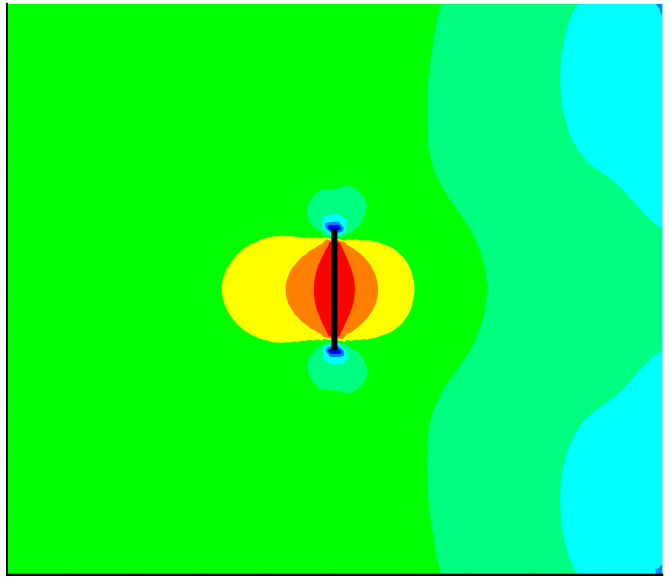

(a)

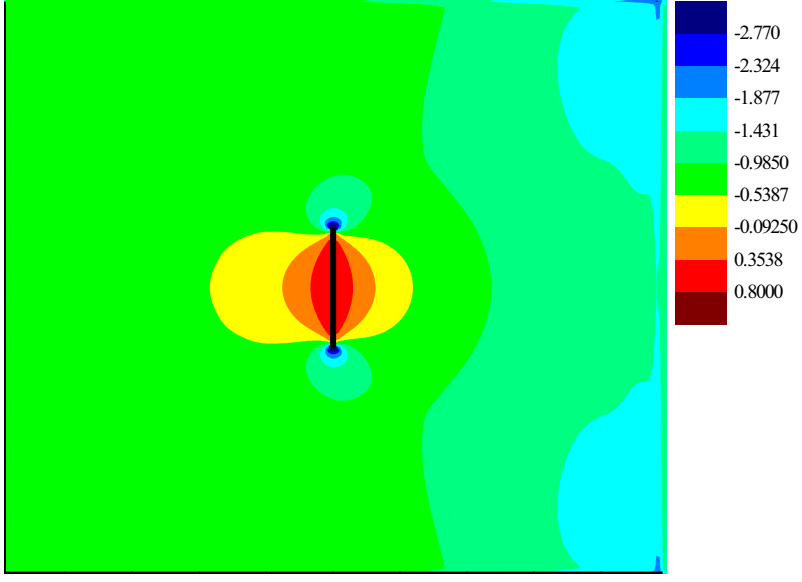

(b)

Fig. 20 Temperature change distributions (K) a ANSYS and b PD results at $t=30 \mu \mathrm{s}$

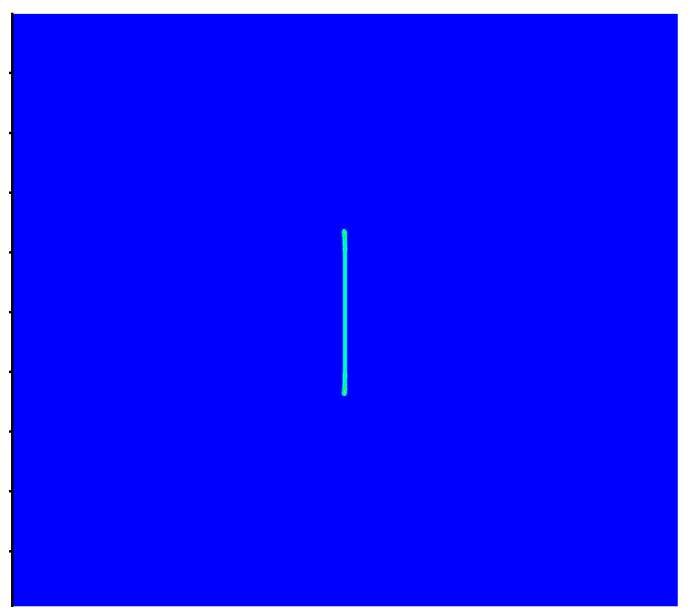

(a)

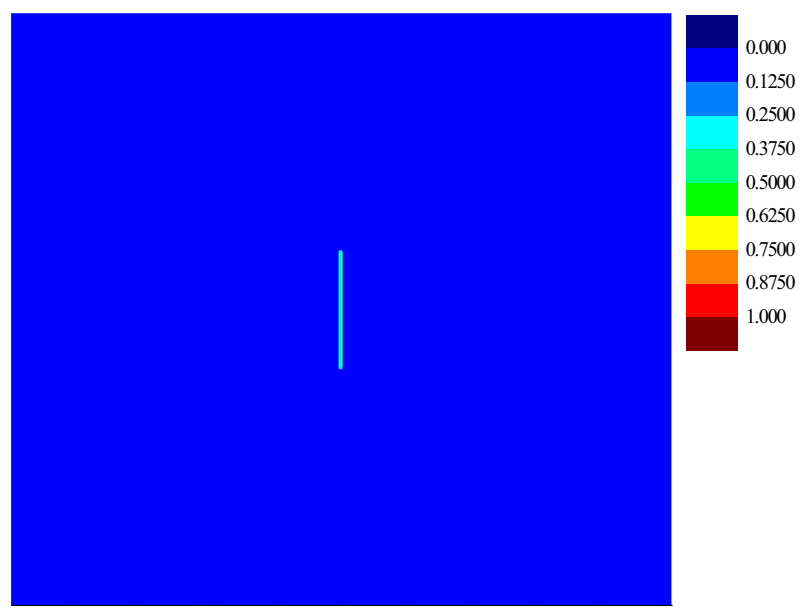

(b)

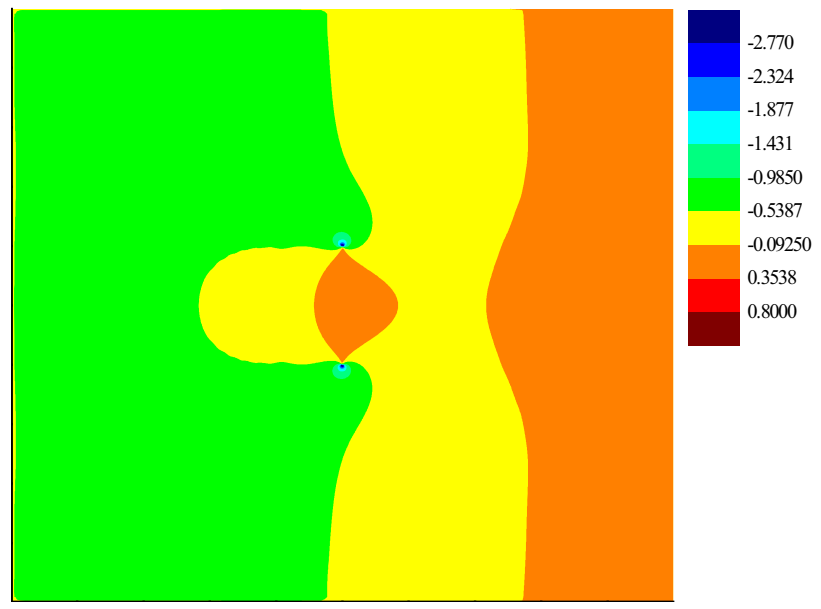

(c)

Fig. 21 Damage plots for a uncoupled case, $\mathbf{b}$ coupled case and $\mathbf{c}$ corresponding temperature change (K) distributions at $t=16 \mu \mathrm{s}$ 


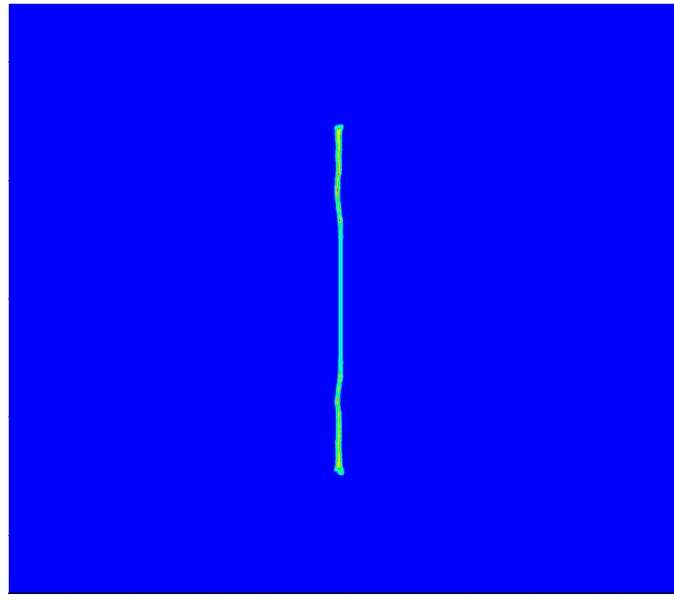

(a)

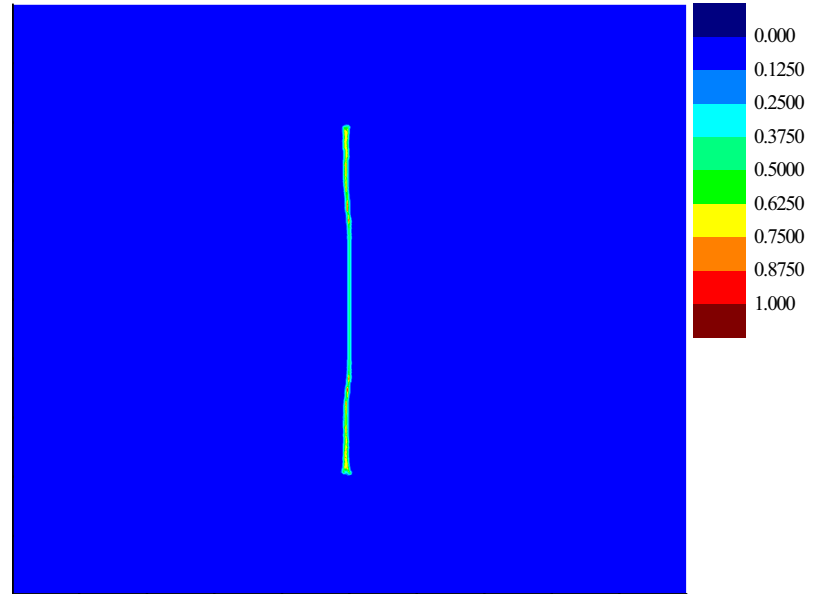

(b)

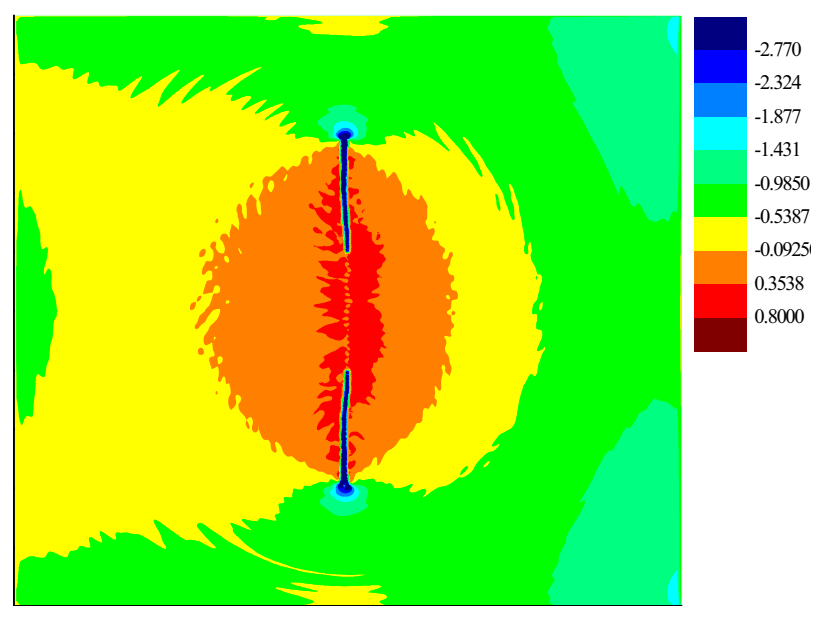

(c)

Fig. 22 Damage plots for a uncoupled case, $\mathbf{b}$ coupled case and $\mathbf{c}$ corresponding temperature change (K) distributions at $t=28 \mu \mathrm{s}$

Figures 18, 19 and 20 present the induced temperature distributions due to applied loading. It is observed that the peridynamic results agree very well with ANSYS solutions. The temperature distributions along $x$-axis propagate almost uniformly in the vertical direction before the thermal wave reaches the crack, as shown in Fig. 18. After the thermal wave reaches or passes the crack, thermal waves get disturbed by the existence of the crack. Higher temperature drop is observed near the crack tip as can be seen from Figs. 19 and 20. This also indicates the stress concentration near the crack tips. Besides, the region near the crack tips is under tension, indicating a tendency of crack growing in the vertical direction. The crack surfaces experience local compression. Therefore, temperature rise is observed in these regions as it can be seen from Fig. 20.

After verification of temperature and deformation field for a plate with a pre-existing crack, as a next example, crack propagation is allowed. The crack configurations at different time steps are provided in Figs. 21, 22 and 23. Crack propagation patterns are compared with coupled and uncoupled cases. Temperature distributions at corresponding stages from coupled cases are also provided.

In the early stage, the cracks grow in similar patterns for both simulation cases. Crack starts to propagate earlier for the uncoupled case (Fig. 21). Cracks both begin to propagate at around $16 \mu \mathrm{s}$. Up to $28 \mu \mathrm{s}$, the cracks propagate in a self-similar manner for both coupled and uncoupled cases. Cracks start branching at around $28 \mu$ s (Fig. 22) and split into visible asymmetrical branches (Fig. 23). In addition, the branches for uncoupled case grow faster than the coupled case.

For coupled case, it is clear that before $16 \mu$ s the temperature distribution is the same with the one obtained from the simulation without crack propagation. However, temperature drops at the crack tips move as the crack 


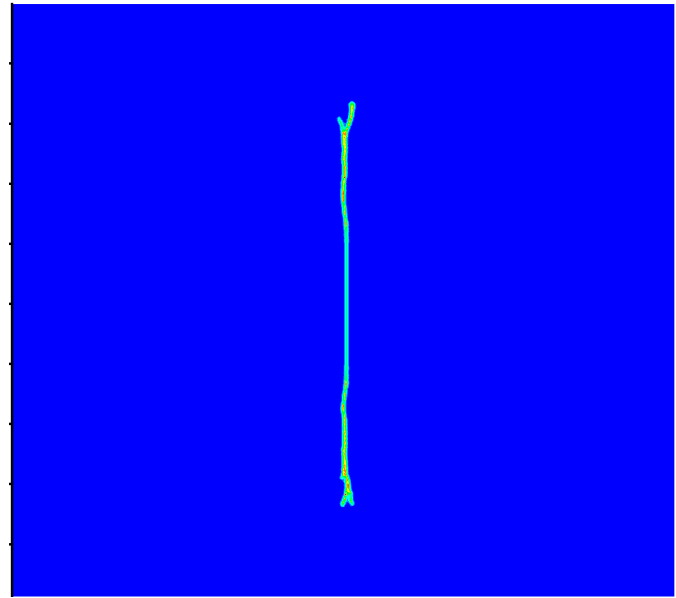

(a)

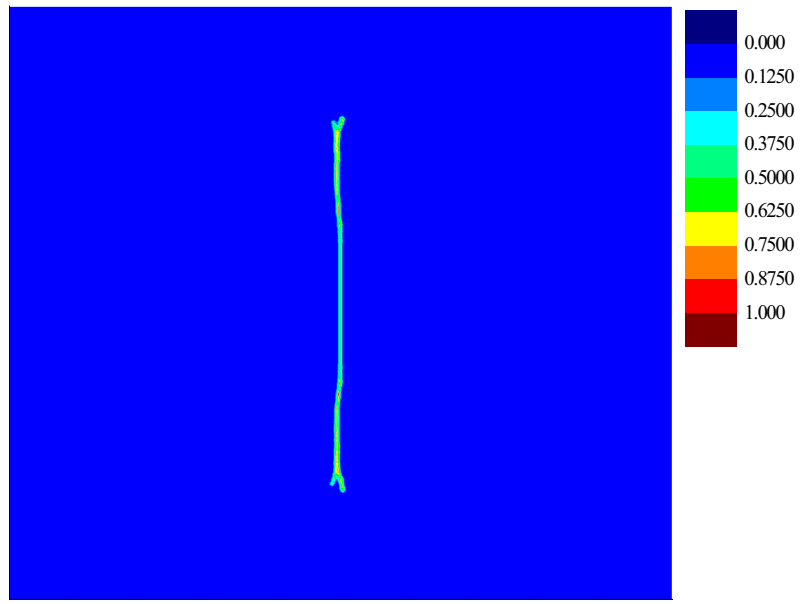

(b)

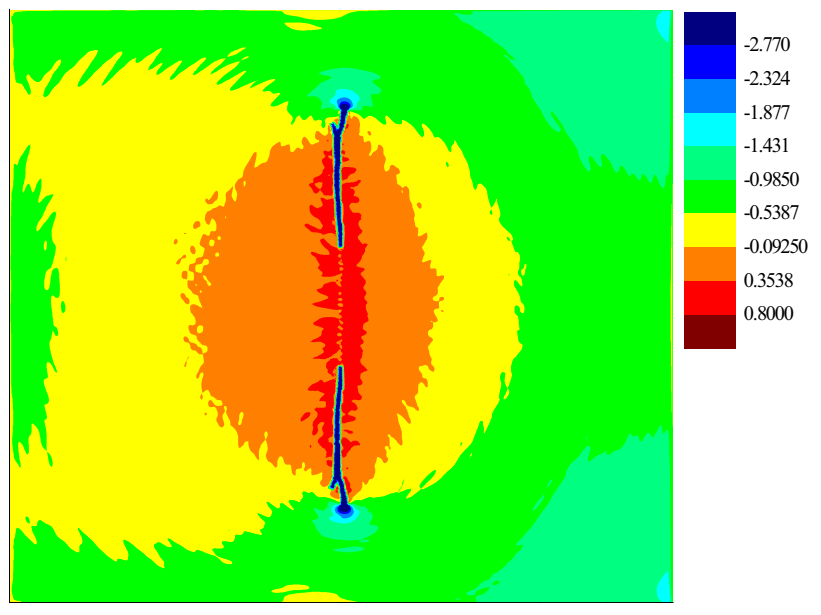

(c)

Fig. 23 Damage plots for a uncoupled case, $\mathbf{b}$ coupled case and $\mathbf{c}$ corresponding temperature change (K) distributions at $t=30 \mu \mathrm{s}$

propagates (Figs. 22c, 23c). The cooling region at the crack tips creates temperature-induced local compression at these regions. Furthermore, the temperature rise around the crack surfaces creates local tension against the compression created by the pressure shock loading. In conclusion, the induced temperature change due to deformation influences the crack growth in the opposite direction against the applied mechanical loading, leading to a reduced degree of crack propagation response. Hence, different crack pattern from uncoupled simulation is obtained.

In conclusion, if shock loadings are applied, large strain rates are created, and thus the coupling term should be considered for more accurate crack propagation predictions.

\subsection{Kalthoff problem simulation}

Kalthoff and Winkler [57] and Kalthoff [55,58] performed a series of experiments where pre-notched plates were subjected to dynamic shear loads. In the experiments, a cylindrical projectile impacted on the notched side of the plate with a constant velocity $\mathbf{V}_{0}$ parallel to the axis of the notch. The pre-existing crack in the upper half steel plate was observed to grow in an angle of approximately $70^{\circ}$ counter clockwise with the notch axis. The failure is in a brittle fracture mode under lower strain rate. These experiments have been successfully simulated by numerical methods such as phase-field simulation [59], finite element method [60]. In addition, 


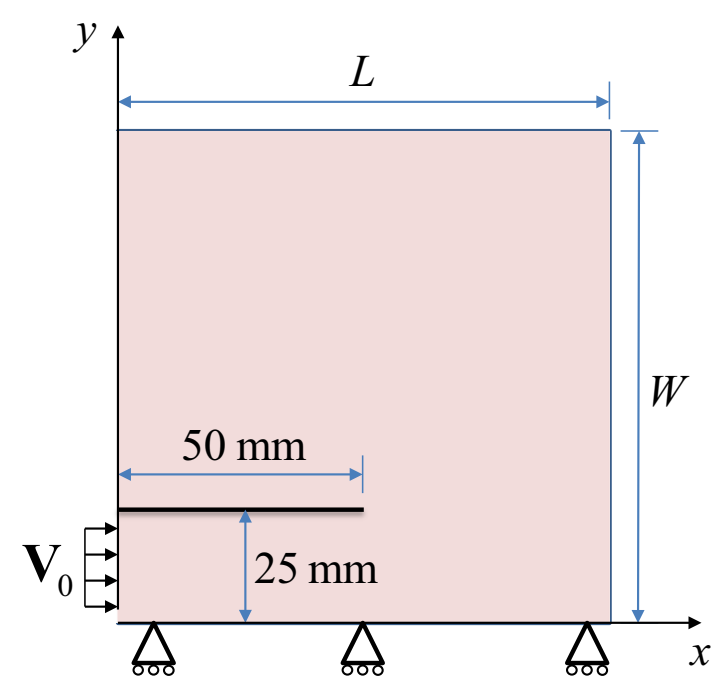

Fig. 24 The geometry and boundary conditions for Kalthoff problem

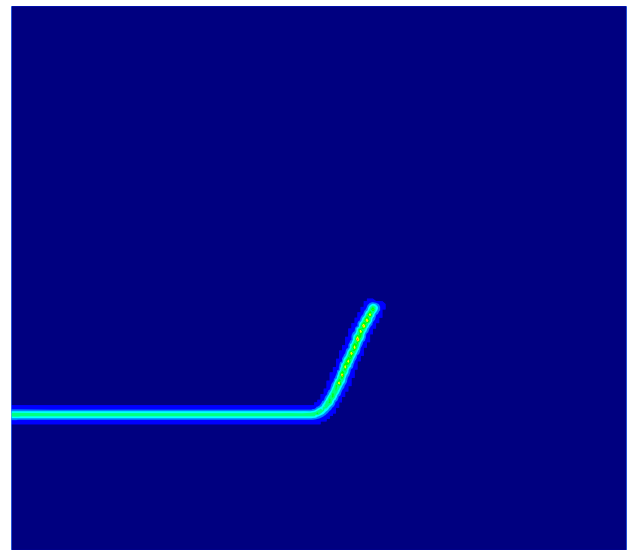

(a)

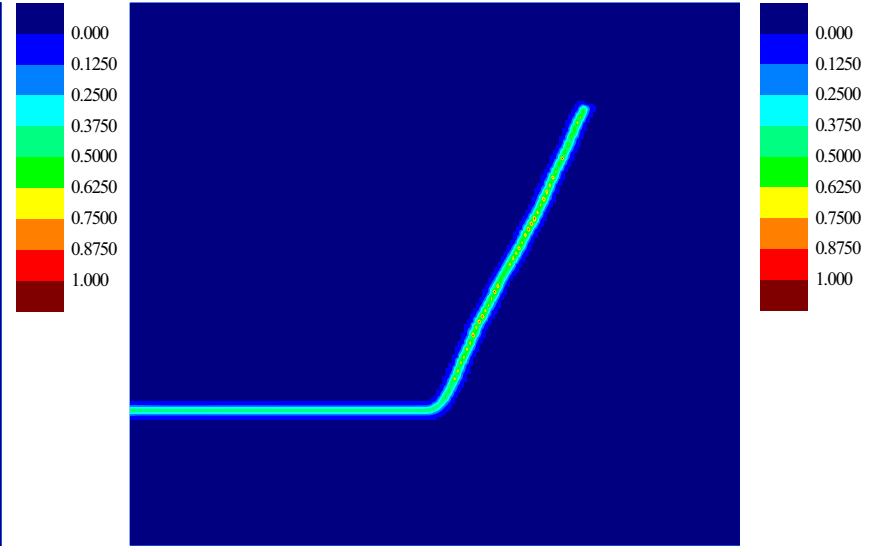

(b)
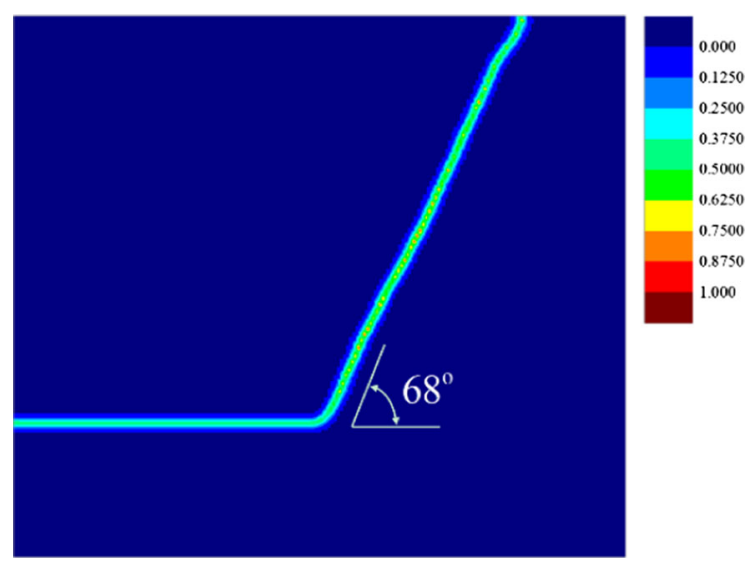

(c)

Fig. 25 Crack evolution at different times, a $t=40 \mu \mathrm{s} ; \mathbf{b} t=65 \mu \mathrm{s} ; \mathbf{c} t=90 \mu \mathrm{s}$ 


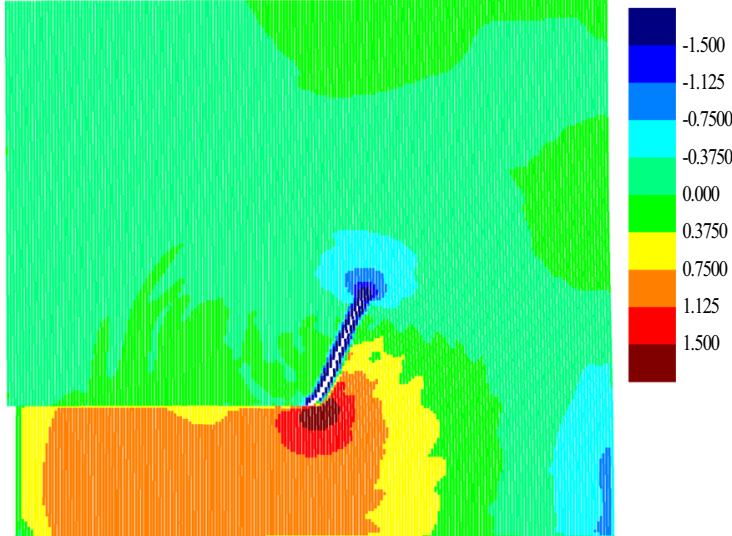

(a)

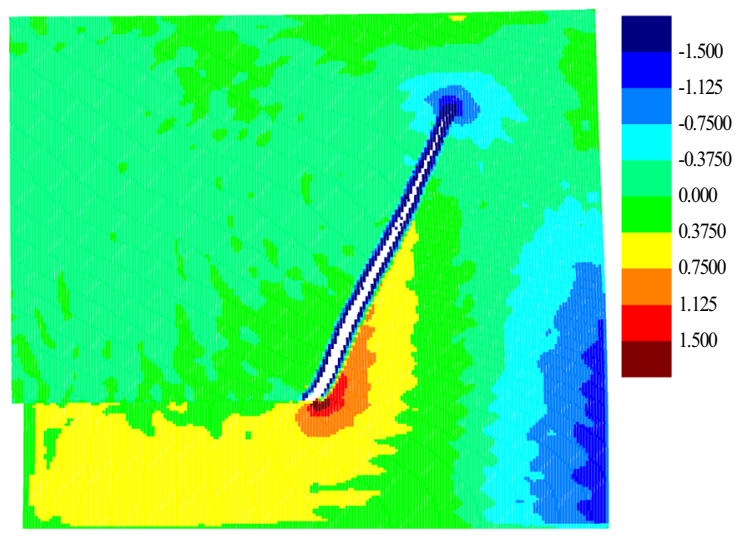

(b)

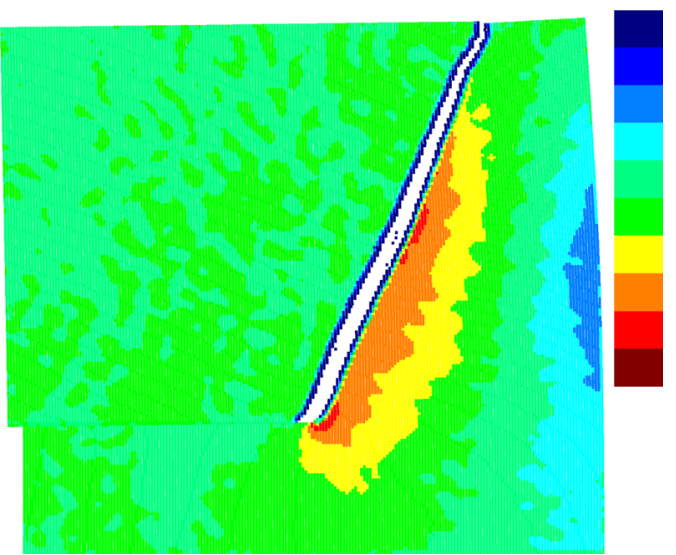

(c)

Fig. 26 Temperature change (K) distributions at different times (displacement scale factor is 3), a $t=40 \mu \mathrm{s} ; \mathbf{b} t=65 \mu \mathrm{s}$; c $t=90 \mu \mathrm{s}$

Silling [61] and Dipasquale et al. [62] used PD to numerically simulate the Kalthoff problem within the realm of mechanical field. In this section, a fully coupled thermomechanical simulation is conducted based on the Kalthoff experiment.

The problem is symmetric so that only the upper half plate is modelled. As shown in Fig. 24, a square plate is modelled with $L=W=100 \mathrm{~mm}$ and its thickness is $1 \mathrm{~mm}$. A pre-existing crack of length being $50 \mathrm{~mm}$ is located above the $x$-axis with the distance of $25 \mathrm{~mm}$. Due to the symmetric conditions, the lower horizontal edge of the plate is fixed in the $y$ direction, i.e. $u_{y}(x, y=0, t)=0$. The other boundaries are free. All the boundaries are thermally insulated. The impact is simulated by imposing a constant velocity to the nodes on the left surface between the crack and the lower horizontal boundary in the PD model. The velocity is parallel to the $x$ direction, and its magnitude is

$$
\left|\mathbf{v}_{0}\right|= \begin{cases}\left(t / t_{0}\right) & v_{0} t \leq t_{0} \\ v_{0} & t>t_{0}\end{cases}
$$

where $\left|\mathbf{v}_{0}\right|$ represents the magnitude of the applied velocity with $v_{0}=16.5 \mathrm{~m} / \mathrm{s}$ and $t_{0}=1 \mu \mathrm{s}$ [59]. The properties of the elastic material are $E=190 \mathrm{GPa}, \rho=8000 \mathrm{~kg} / \mathrm{m}^{3}, v=0.3, c_{\mathrm{v}}=477 \mathrm{~J} /(\mathrm{kgK})$, $\alpha=17.6 \times 10^{-6} \mathrm{~K}^{-1}, k_{\mathrm{T}}=16.2 \mathrm{~W} /(\mathrm{mK})$. The critical energy release rate is $G_{\mathrm{c}}=2.213 \times 10^{4} \mathrm{~J} / \mathrm{m}^{2}$. The reference temperature is $\Theta_{0}=285 \mathrm{~K}$. As to the PD discretization, the mesh size is $\Delta x=0.0005 \mathrm{~m}$ and the horizon is chosen as $\delta=3.015 \Delta x$. The time step size is $0.01 \mu \mathrm{s}$, and the total simulation time is $90 \mu \mathrm{s}$. The critical stretch value is calculated as $s_{\mathrm{c}}=0.0103$.

The crack is observed to propagate at $t=20 \mu \mathrm{s}$. The crack evolution at different times is provided in Fig. 25. The angle between the crack path and the positive $x$ direction is observed to be $68^{\circ}$, which is close to the 


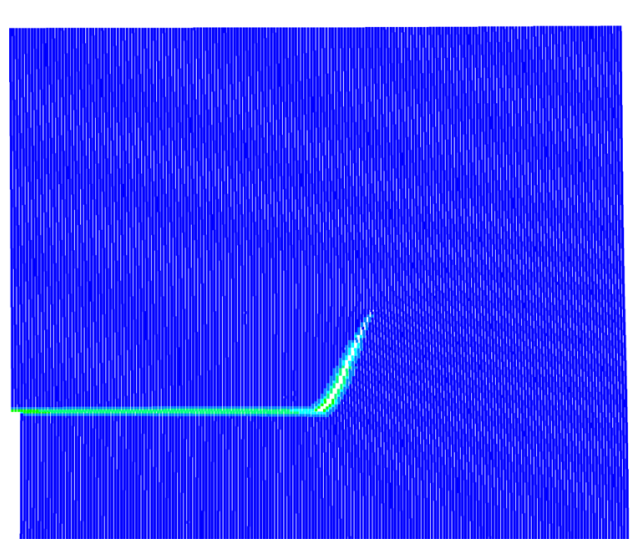

(a)
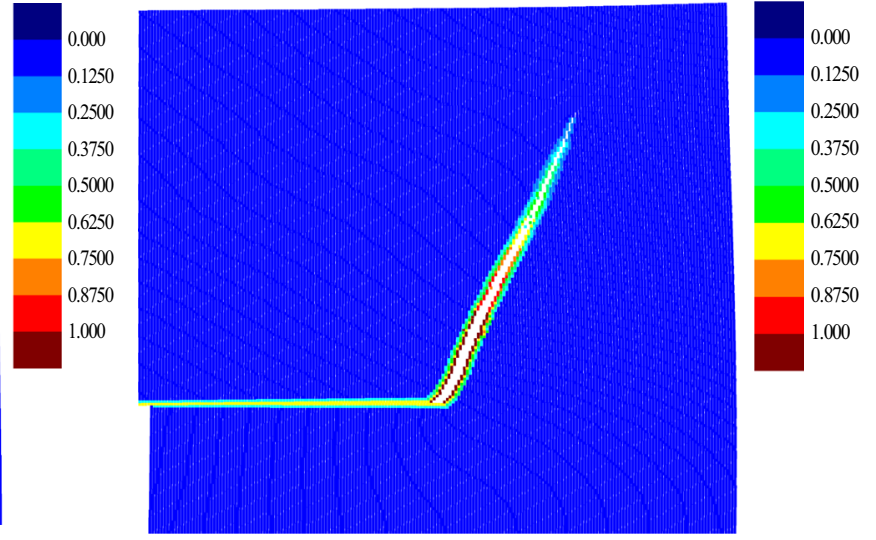

(b)

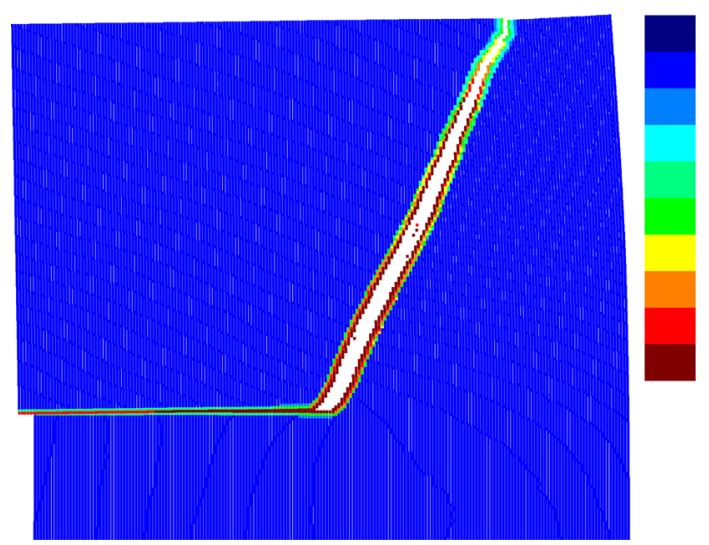

(c)

Fig. 27 Maximum stretch distributions in deformed configurations (displacement scale factor is 3) at different times, $\mathbf{a} t=40 \mu \mathrm{s}$; $\mathbf{b} t=65 \mu \mathrm{s} ; \mathbf{c} t=90 \mu \mathrm{s}$

corresponding result of the Kalthoff experiment. Furthermore, the temperature change distribution evolutions are provided in Fig. 26. The maximum stretch distributions in deformed configurations are presented in Fig. 27. It can be observed temperature rises near the crack and temperature drops in the crack, which agrees with the conclusions drawn in the last two simulation cases.

\section{Conclusion}

In this study, fully coupled thermoelastic equations in ordinary state-based peridynamic theory are provided, including their non-dimensional forms. To verify the PD model, some benchmark problems are solved by using both peridynamics and FEM solutions. The good agreement between PD and other methods indicates the validity of the proposed PD model. Finally, crack propagation patterns are predicted for three-point bending test, Kalthoff problem and a plate with a pre-existing crack subjected to a pressure load. The following conclusions are drawn

1. The ordinary state-based fully coupled thermomechanical PD model introduced in this paper is capable of predicting temperature and displacement responses accurately both for dimensional and for nondimensional problems.

2. When shock loadings are applied, the coupling effect on displacements and temperature should be taken into consideration for more accurate results.

3. The coupling terms do have an effect on crack propagation when shock loadings are applied. Therefore, fully coupled analysis is necessary in such cases. 
Acknowledgements The authors gratefully acknowledge financial support from China Scholarship Council (CSC No. 201506230126) and University of Strathclyde.

Open Access This article is distributed under the terms of the Creative Commons Attribution 4.0 International License (http:// creativecommons.org/licenses/by/4.0/), which permits unrestricted use, distribution, and reproduction in any medium, provided you give appropriate credit to the original author(s) and the source, provide a link to the Creative Commons license, and indicate if changes were made.

\section{Appendix A}

A.1 Ordinary state-based thermal modulus for a material point

The thermal modulus for a material point can be obtained by using the PD local thermal modulus formulation provided in Eq. (21) as follows:

When small deformation approximation is adopted, $\Lambda$ provided in Eq. (9) results in $\Lambda=1$. Consequently, the integration term in Eq. (21) becomes

$$
\int_{H_{\mathbf{x}}} \Lambda \mathrm{d} V^{\prime}=V_{H_{\mathbf{x}}}
$$

with $V_{H_{\mathbf{x}}}$ representing the integration volume.

For 2D problems, the integration domain is a circle disc with its radius, $\delta$, and thickness, $h$. On the other hand, the integration domain for 3D problems is a sphere; thus, the volume in Eq. (A.1) can be calculated as

$$
\begin{aligned}
& V_{H_{\mathbf{x}}}=\pi h \delta^{2} \text { for } 2 \mathrm{D} \\
& V_{H_{\mathbf{x}}}=\frac{4 \pi \delta^{3}}{3} \text { for } 3 \mathrm{D}
\end{aligned}
$$

An average value of relative position can also be evaluated by using weighted integration as

$$
\begin{aligned}
|\xi|_{\text {avg }}= & \frac{\int_{H_{\mathbf{x}}}|\xi| \mathrm{d} V^{\prime}}{\int_{H_{\mathbf{x}}} \mathrm{d} V^{\prime}}=\frac{\int_{0}^{2 \pi} \int_{0}^{\delta}|\xi| h|\xi| \mathrm{d}|\xi| \mathrm{d} \vartheta}{V_{H_{\mathbf{x}}}}=\frac{2 \pi h \delta^{3} / 3}{\pi h \delta^{2}}=\frac{2 \delta}{3} \text { for } 2 \mathrm{D} \\
|\xi|_{\text {avg }}= & \frac{\int_{H_{\mathbf{x}}}|\xi| \mathrm{d} V^{\prime}}{\int_{H_{\mathbf{x}}} \mathrm{d} V^{\prime}}=\frac{\iint_{0}^{\delta} \int_{0}^{2 \pi} \int_{0}^{\pi}|\xi||\xi|^{2} \sin (\phi) d \phi \mathrm{d} \vartheta \mathrm{d}|\xi|}{V_{H_{\mathbf{x}}}}=\frac{\pi \delta^{4}}{4 \pi \delta^{3} / 3}=\frac{3 \delta}{4} \text { for } 3 \mathrm{D}
\end{aligned}
$$

with $\xi=\mathbf{x}^{\prime}-\mathbf{x}$ (Fig. 1). In Eq. (A.3) $\vartheta$ represents the bond angle with respect to $x$-axis and $\phi$ represents the bond angle with respect to the $x-y$ plane for $3 \mathrm{D}$ case

Recalling the classical material constants

$$
\begin{aligned}
& K_{\theta}=\frac{E}{2(1-v)}, \mu=\frac{E}{2(1+v)} \text { for } 2 \mathrm{D} \\
& K_{\theta}=\frac{E}{3(1-2 v)}, \mu=\frac{E}{2(1+v)} \text { for } 3 \mathrm{D}
\end{aligned}
$$

Finally, by substituting Eqs. (A.2), (A.3) and Eq. (A.4) into (18), average PD thermal modulus for a material point can be calculated as

$$
\begin{aligned}
& \bar{\beta}=\frac{3 E \alpha}{\pi h \delta^{3}(1-v)} \text { for } 2 \mathrm{D} \\
& \bar{\beta}=\frac{3 E \alpha}{\pi \delta^{4}(1-2 \nu)} \text { for } 3 \mathrm{D}
\end{aligned}
$$


PD thermal modulus for ordinary state-based form reduces to bond-based form by plugging in $v=1 / 3$ for $2 \mathrm{D}$ and $v=1 / 4$ for $3 \mathrm{D}$ in Eq. (A.5) [43] as

$$
\beta_{b}=\frac{c \alpha}{2}
$$

with

$$
c=\left\{\begin{array}{c}
\frac{2 E}{A \delta^{2}} \text { for } 1 \mathrm{D} \\
\frac{9 E}{\pi h \delta^{3}} \text { for } 2 \mathrm{D} \text { with } v=\frac{1}{3} \\
\frac{12 E}{\pi \delta^{4}} \text { for } 3 \mathrm{D} \text { with } v=\frac{1}{4}
\end{array}\right.
$$

As it can be seen from the above formulations, bond-based PD has a limitation on Poisson's ratio [48].

\section{A.2 Bond-based PD form of thermomechanical equations}

\section{Equation of motion}

In bond-based PD by applying the restriction $a=0$ in Chapter 4 of [49], the PD auxiliary parameters A and B provided in Eqs. (4) and (5) become

$$
\begin{aligned}
& A=4 \delta b(s-\alpha T) \\
& B=4 \delta b\left(s-\alpha T^{\prime}\right)
\end{aligned}
$$

Corresponding PD force functions provided in Eqs. (2) and (3) become

$$
\begin{aligned}
\mathbf{t}\left(\mathbf{u}^{\prime}-\mathbf{u}, \mathbf{x}^{\prime}-\mathbf{x}, t\right) & =2 \delta b(s-\alpha T) \frac{\mathbf{y}^{\prime}-\mathbf{y}}{\left|\mathbf{y}^{\prime}-\mathbf{y}\right|} \\
\mathbf{t}^{\prime}\left(\mathbf{u}-\mathbf{u}^{\prime}, \mathbf{x}-\mathbf{x}^{\prime}, t\right) & =-2 \delta b\left(s-\alpha T^{\prime}\right) \frac{\mathbf{y}^{\prime}-\mathbf{y}}{\left|\mathbf{y}^{\prime}-\mathbf{y}\right|}
\end{aligned}
$$

By enforcing Eqs. (A.8a) and (A.8b) to be equal to each other as

$$
\begin{aligned}
\mathbf{t}\left(\mathbf{u}^{\prime}-\mathbf{u}, \mathbf{x}^{\prime}-\mathbf{x}, t\right) & =2 \delta b\left(s-\alpha \frac{\left(T+T^{\prime}\right)}{2}\right) \frac{\mathbf{y}^{\prime}-\mathbf{y}}{\left|\mathbf{y}^{\prime}-\mathbf{y}\right|} \\
\mathbf{t}^{\prime}\left(\mathbf{u}-\mathbf{u}^{\prime}, \mathbf{x}-\mathbf{x}^{\prime}, t\right) & =-2 \delta b\left(s-\alpha \frac{\left(T+T^{\prime}\right)}{2}\right) \frac{\mathbf{y}^{\prime}-\mathbf{y}}{\left|\mathbf{y}^{\prime}-\mathbf{y}\right|}
\end{aligned}
$$

which results in $\mathbf{t}=-\mathbf{t}^{\prime}$. By plugging in Eqs. (A.9a) and (A.9b) into Eq. (1), the equation of motion becomes [43]

$$
\rho \ddot{\mathbf{u}}(\mathbf{x}, t)=\int_{H_{\mathbf{x}}}\left(4 \delta b s-2 b \delta \alpha\left(T+T^{\prime}\right)\right)\left(\frac{\mathbf{y}^{\prime}-\mathbf{y}}{\left|\mathbf{y}^{\prime}-\mathbf{y}\right|}\right) \mathrm{d} V^{\prime}+\mathbf{b}(\mathbf{x}, t)
$$

or

$$
\rho \ddot{\mathbf{u}}(\mathbf{x}, t)=\int_{H_{\mathbf{x}}}\left(c s-\beta_{b}\left(T+T^{\prime}\right)\right)\left(\frac{\mathbf{y}^{\prime}-\mathbf{y}}{\left|\mathbf{y}^{\prime}-\mathbf{y}\right|}\right) \mathrm{d} V^{\prime}+\mathbf{b}(\mathbf{x}, t)
$$

with bond-based thermal modulus as

$$
\beta_{b}=2 b \alpha
$$

or

$$
\beta_{b}=\frac{c \alpha}{2}
$$


with $c$ expressed in Eq.(A.6b).

\section{Heat equation}

The form of heat equation in bond-based peridynamics remains the same as Eq. (12) [43]

$$
\rho c_{\mathrm{v}} \dot{T}(\mathbf{x}, t)=\int_{H_{\mathbf{x}}}\left(\kappa \frac{\Theta\left(\mathbf{x}^{\prime}, t\right)-\Theta(\mathbf{x}, t)}{\left|\mathbf{x}^{\prime}-\mathbf{x}\right|}-\Theta_{0} \beta_{b} \dot{e}\left(\mathbf{x}^{\prime}-\mathbf{x}\right)\right) \mathrm{d} V^{\prime}+h_{s}(\mathbf{x}, t)
$$

with bond-based thermal modulus provided in Eq. (A.10)

\section{References}

1. Spitsberg, I., Steibel, J.: Thermal and environmental barrier coatings for SiC/Sic CMCs in aircraft engine applications. Int. J. Appl. Ceram. Technol. 1(4), 291-301 (2004)

2. Nali, P., Carrera, E., Calvi, A.: Advanced fully coupled thermo-mechanical plate elements for multilayered structures subjected to mechanical and thermal loading. Int. J. Numer. Meth. Eng. 85(7), 896-919 (2011)

3. Biot, M.A.: Thermoelasticity and irreversible thermodynamics. J. Appl. Phys. 27(3), 240-253 (1956)

4. Herrmann, G.: On variational principles in thermoelasticity and heat conduction. Q. Appl. Math. 21(2), 151-155 (1963)

5. Jabbari, M., Dehbani, H., Eslami, M.R.: An exact solution for classic coupled thermoelasticity in spherical coordinates. J. Press. Vessel Technol. 132(3), 031201-031211 (2010)

6. Jabbari, M., Dehbani, H., Eslami, M.R.: An exact solution for classic coupled thermoelasticity in cylindrical coordinates. J. Press. Vessel Technol. 133(5), 051204-051210 (2011)

7. Dillon, O., Tauchert, T.: The experimental technique for observing the temperatures due to the coupled thermoelastic effect. Int. J. Solids Struct. 2(3), 385-391 (1966)

8. Abali, B.E.: Computational Reality: Solving Nonlinear and Coupled Problems in Continuum Mechanics, vol. 55. Springer, New York (2016)

9. Cannarozzi, A.A., Ubertini, F.: A mixed variational method for linear coupled thermoelastic analysis. Int. J. Solids Struct. 38(4), 717-739 (2001)

10. Tehrani, P.H., Eslami, M.R.: Boundary element analysis of coupled thermoelasticity with relaxation times in finite domain. AIAA J. 38(3), 534-541 (2000)

11. Atkinson, C., Craster, R.V.: Fracture in fully coupled dynamic thermoelasticity. J. Mech. Phys. Solids 40(7), 1415-1432 (1992)

12. Weichert, R., Schönert, K.: Heat generation at the tip of a moving crack. J. Mech. Phys. Solids 26(3), 151-161 (1978)

13. Bhalla, K.S., Zehnder, A.T., Han, X.: Thermomechanics of slow stable crack growth: closing the loop between experiments and computational modeling. Eng. Fract. Mech. 70(17), 2439-2458 (2003)

14. Miehe, C., Schanzel, L.M., Ulmer, H.: Phase field modeling of fracture in multi-physics problems. Part I. Balance of crack surface and failure criteria for brittle crack propagation in thermo-elastic solids. Comput. Method Appl. Mech. 294, 449-485 (2015)

15. Silling, S.A.: Reformulation of elasticity theory for discontinuities and long-range forces. J. Mech. Phys. Solids 48(1), $175-209(2000)$

16. Dell'Isola, F., Ugo, A., Luca, P.: At the origins and in the vanguard of peridynamics, non-local and higher-gradient continuum mechanics: an underestimated and still topical contribution of Gabrio Piola. Math. Mech. Solids. 20(8), 887-928 (2015)

17. Eugster, S.R., Dell'Isola, F.: Exegesis of the introduction and Sect. I from Fundamentals of the Mechanics of Continua by E. Hellinger. ZAMM - Zeitschrift für Angewandte Mathematik und Mechanik. 97(4), 477-506 (2017)

18. Eugster, S.R., Dell'Isola, F.: Exegesis of Sect. II and III.A from Fundamentals of the Mechanics of Continua by E. Hellinger. ZAMM - Journal of Applied Mathematics and Mechanics / Zeitschrift für Angewandte Mathematik und Mechanik. (2017). https://doi.org/10.1002/zamm.201600293

19. Eugster, S.R., Dell'Isola, F.: Exegesis of Sect. III.B from Fundamentals of the Mechanics of Continua by E. Hellinger. ZAMM - Journal of Applied Mathematics and Mechanics / Zeitschrift für Angewandte Mathematik und Mechanik. 98(1), 69-105 (2017)

20. Dell'Isola, F., Alessandro, D.C., Ivan, G.: Higher-gradient continua: the legacy of Piola, Mindlin, Sedov and Toupin and some future research perspectives. Math. Mech. Solids. (2016). https://doi.org/10.1177/1081286515616034

21. Dell'Isola, F., Alessandro D.C., Raffaele, E., Lucio, R.: Some cases of unrecognized transmission of scientific knowledge: from antiquity to gabrio piola's peridynamics and generalized continuum theories. In: Generalized continua as models for classical and advanced materials, vol. 42, pp 77-128. Springer, Cham (2016)

22. Demmie, P.N., Silling, S.A.: An approach to modeling extreme loading of structures using peridynamics. J. Mech. Mater. Struct. 2(10), 1921-1945 (2007)

23. Silling, S., Weckner, O., Askari, E., Bobaru, F.: Crack nucleation in a peridynamic solid. Int. J. Fract. 162(1-2), 219-227 (2010)

24. Ha, Y.D., Bobaru, F.: Characteristics of dynamic brittle fracture captured with peridynamics. Eng. Fract. Mech. 78(6), $1156-1168(2011)$

25. Huang, D., Lu, G.D., Qiao, P.Z.: An improved peridynamic approach for quasi-static elastic deformation and brittle fracture analysis. Int. J. Mech. Sci. 94-95, 111-122 (2015)

26. Hu, W.K., Ha, Y.D., Bobaru, F.: Peridynamic model for dynamic fracture in unidirectional fiber-reinforced composites. Comput. Method Appl. Mech. 217, 247-261 (2012) 
27. Bobaru, F., Hu, W.K.: The meaning, selection, and use of the peridynamic horizon and its relation to crack branching in brittle materials. Int. J. Fract. 176(2), 215-222 (2012)

28. Agwai, A., Guven, I., Madenci, E.: Predicting crack propagation with peridynamics: a comparative study. Int. J. Fract. 171(1), 65-78 (2011)

29. Vazic, B., Wang, H., Diyaroglu, C., Oterkus, S., Oterkus, E.: Dynamic propagation of a macrocrack interacting with parallel small cracks. AIMS Mater. Sci. 4(1), 118-136 (2017)

30. Wang, H., Oterkus, E., Celik, S., Toros, S.: Thermomechanical analysis of porous solid oxide fuel cell by using peridynamics. AIMS Energy 5(4), 585-600 (2017)

31. Oterkus, S., Madenci, E., Oterkus, E., Hwang, Y., Bae, J., Han, S.: Hygro-thermo-mechanical analysis and failure prediction in electronic packages by using peridynamics. In: 2014 IEEE 64th Electronic Components and Technology Conference (ECTC), pp. 973-982. IEEE (2014

32. Amani, J., Oterkus, E., Areias, P., Zi, G., Nguyen-Thoi, T., Rabczuk, T.: A non-ordinary state-based peridynamics formulation for thermoplastic fracture. Int. J. Impact Eng. 87, 83-94 (2016)

33. Diyaroglu, C., Oterkus, E., Madenci, E., Rabczuk, T., Siddiq, A.: Peridynamic modeling of composite laminates under explosive loading. Compos. Struct. 144, 14-23 (2016)

34. Diyaroglu, C., Oterkus, E., Oterkus, S., Madenci, E.: Peridynamics for bending of beams and plates with transverse shear deformation. Int. J. Solids Struct. 69, 152-168 (2015)

35. Oterkus, E., Madenci, E.: Peridynamics for failure prediction in composites. In: 53rd AIAA/ASME/ASCE/AHS/ASC Structures, Structural Dynamics and Materials Conference 20th AIAA/ASME/AHS Adaptive Structures Conference 14th AIAA. p. $1692(2012)$

36. Oterkus, S., Madenci, E.: Peridynamics for antiplane shear and torsional deformations. J. Mech. Mater. Struct 10, 167-193 (2015)

37. Oterkus, S., Fox, J., Madenci, E.: Simulation of electro-migration through peridynamics. In: 2013 IEEE 63rd Electronic Components and Technology Conference (ECTC). pp. 1488-1493. IEEE (2013)

38. Oterkus, S., Madenci, E., Oterkus, E.: Fully coupled poroelastic peridynamic formulation for fluid-filled fractures. Eng. Geol. 225, 19-28 (2017)

39. Oterkus, S., Madenci, E., Agwai, A.: Peridynamic thermal diffusion. J. Comput. Phys. 265, 71-96 (2014)

40. Oterkus, S., Madenci, E.: Peridynamic modeling of fuel pellet cracking. Eng. Fract. Mech. 176, 23-37 (2017)

41. Bobaru, F., Duangpanya, M.: A peridynamic formulation for transient heat conduction in bodies with evolving discontinuities. J. Comput. Phys. 231(7), 2764-2785 (2012)

42. Bobaru, F., Duangpanya, M.: The peridynamic formulation for transient heat conduction. Int. J. Heat Mass Transf. 53(19-20), 4047-4059 (2010)

43. Oterkus, S., Madenci, E., Agwai, A.: Fully coupled peridynamic thermomechanics. J. Mech. Phys. Solids 64, 1-23 (2014)

44. Oterkus, S.: Peridynamics for the Solution of Multiphysics Problems. The University of Arizona, Tucson (2015)

45. Madenci, E., Oterkus, S.: Peridynamics for coupled field equations. In: Bobaru, F., Foster, J.T., Geubelle, P.H., Silling, S.A. (eds.) Handbook of Peridynamic Modeling, pp. 489-531. Fl, Boca Raton (2016)

46. Oterkus, S., Madenci, E.: Fully coupled thermomechanical analysis of fiber reinforced composites using peridynamics. In: 55th AIAA/ASMe/ASCE/AHS/SC Structures, Structural Dynamics, and Materials Conference-SciTech Forum and Exposition, Maryland (2014)

47. Oterkus, S., Madenci, E.: Crack growth prediction in fully-coupled thermal and deformation fields using peridynamic theory. In: 54th AIAA Structures, Structural Dynamics and Materials Conference, Boston, Massachusetts (2013)

48. Silling, S.A., Askari, E.: A meshfree method based on the peridynamic model of solid mechanics. Comput. Struct. 83(17), $1526-1535$ (2005)

49. Madenci, E., Oterkus, E.: Peridynamic Theory and Its Applications. Springer, New York (2014)

50. Agwai, A.G.: A peridynamic approach for coupled fields. The University of Arizona, Tucson (2011)

51. Silling, S.A.: Linearized theory of peridynamic states. J. Elast. 99(1), 85-111 (2010)

52. Lord, H.W., Shulman, Y.: A generalized dynamical theory of thermoelasticity. J. Mech. Phys. Solids 15(5), 299-309 (1967)

53. Sackman, J.: Approximate solutions in linear, coupled thermoelasticity. J. Appl. Mech. 35, 255-266 (1968)

54. Hosseini-Tehrani, P., Eslami, M.R.: BEM analysis of thermal and mechanical shock in a two-dimensional finite domain considering coupled thermoelasticity. Eng. Anal. Bound Elem. 24(3), 249-257 (2000)

55. Kalthoff, J.F.: Modes of dynamic shear failure in solids. Int. J. Fract. 101(1), 1-31 (2000)

56. Chadwick, P.: Thermo-mechanics of rubberlike materials. Philos.Trans. R. Soc. Lond. A: Math. Phys. Eng. Sci. 276(1260), 371-403 (1974)

57. Kalthoff, J., Winkler, S.: Failure mode transition at high rates of shear loading. DGM Informations gesellschaft mbH, Impact Loading Dyn. Beh. Mater. 1, 185-195 (1988)

58. Kalthoff, J.F.: Shadow optical analysis of dynamic shear fracture. In: SPIE. p. 6 (1988)

59. Borden, M.J., Verhoosel, C.V., Scott, M.A., Hughes, T.J.R., Landis, C.M.: A phase-field description of dynamic brittle fracture. Comput. Method Appl. Mech. 217-220, 77-95 (2012)

60. Batra, R.C., Ravinsankar, M.V.S.: Three-dimensional numerical simulation of the Kalthoff experiment. Int. J. Fract. 105(2), 161-186 (2000)

61. Silling, S.: Peridynamic modeling of the Kalthoff-Winkler experiment. Submission for the (2001)

62. Dipasquale, D., Zaccariotto, M., Galvanetto, U.: Crack propagation with adaptive grid refinement in 2D peridynamics. Int. J. Fract. 190(1), 1-22 (2014)

Publisher's Note Springer Nature remains neutral with regard to jurisdictional claims in published maps and institutional affiliations. 